\title{
Eight Flurothyl-Induced Generalized Seizures Lead to the Rapid Evolution of Spontaneous Seizures in Mice: A Model of Epileptogenesis with Seizure Remission
}

\author{
[DSridhar B. Kadiyala, ${ }^{1}$ - Joshua Q. Yannix, ${ }^{3,4}$ - Julia W. Nalwalk, ${ }^{1}$ Dominick Papandrea, ${ }^{1,5}$ Barbara S. Beyer, ${ }^{1 \dagger}$ \\ ¿Bruce J. Herron, ${ }^{3,4}$ and $\odot$ Russell J. Ferland ${ }^{1,2}$ \\ Departments of ${ }^{1}$ Neuroscience and Experimental Therapeutics, and ${ }^{2}$ Neurology, Albany Medical College, Albany, New York 12208, ${ }^{3}$ Wadsworth Center, \\ Albany, New York 12201, ${ }^{4}$ Department of Biomedical Sciences, School of Public Health, SUNY Albany, Albany, New York 12201, and ${ }^{5}$ Department of \\ Biology, Center for Biotechnology and Interdisciplinary Studies, Rensselaer Polytechnic Institute, Troy, New York 12180
}

The occurrence of recurrent, unprovoked seizures is the hallmark of human epilepsy. Currently, only two-thirds of this patient population has adequate seizure control. New epilepsy models provide the potential for not only understanding the development of spontaneous seizures, but also for testing new strategies to treat this disorder. Here, we characterize a primary generalized seizure model of epilepsy following repeated exposure to the $\mathrm{GABA}_{\mathrm{A}}$ receptor antagonist, flurothyl, in which mice develop spontaneous seizures that remit within 1 month. In this model, we expose C57BL/6 mice to flurothyl until they experience a generalized seizure. Each of these generalized seizures typically lasts $<30 \mathrm{~s}$. We induce one seizure per day for $8 \mathrm{~d}$ followed by $24 \mathrm{~h}$ video-electroencephalographic recordings. Within $1 \mathrm{~d}$ following the last of eight flurothyl-induced seizures, $\sim 50 \%$ of mice have spontaneous seizures. Ninety-five percent of mice tested have seizures within the first week of the recording period. Of the spontaneous seizures recorded, the majority are generalized clonic seizures, with the remaining $7-12 \%$ comprising generalized clonic seizures that transition into brainstem seizures. Over the course of an 8 week recording period, spontaneous seizure episodes remit after $\sim 4$ weeks. Overall, the repeated flurothyl paradigm is a model of epileptogenesis with spontaneous seizures that remit. This model provides an additional tool in our armamentarium for understanding the mechanisms underlying epileptogenesis and may provide insights into why spontaneous seizures remit without anticonvulsant treatment. Elucidating these processes could lead to the development of new epilepsy therapeutics.

Key words: electroencephalogram; epilepsy; epileptogenesis; flurothyl; remission; valproate

Significance Statement

Epilepsy is a chronic disorder characterized by the occurrence of recurrent, unprovoked seizures in which the individual seizureictal events are self-limiting. Remission of recurrent, unprovoked seizures can be achieved in two-thirds of cases by treatment with anticonvulsant medication, surgical resection, and/or nerve/brain electrode stimulation. However, there are examples in humans of epilepsy with recurrent, unprovoked seizures remitting without any intervention. While elucidating how recurrent, unprovoked seizures develop is critical for understanding epileptogenesis, an understanding of how and why recurrent, unprovoked seizures remit may further our understanding and treatment of epilepsy. Here, we describe a new model of recurrent, unprovoked spontaneous seizures in which the occurrence of spontaneous seizures naturally remits over time without any therapeutic intervention.

\section{Introduction}

Epilepsy is a complex and multifactorial disease defined by recurrent, unprovoked seizures (Szs) that usually are self-limiting (i.e., an exception would be status epilepticus). Seizures are well controlled with anticonvulsant drug regimens in approximately two-

Received Aug. 5, 2014; revised May 26, 2016; accepted May 31, 2016.

Author contributions: S.B.K., D.P., and R.J.F. designed research; S.B.K., J.W.N., D.P., B.S.B., and R.J.F. performed research; S.B.K. and R.J.F. contributed unpublished reagents/analytic tools; S.B.K., J.Q.Y., J.W.N., B.J.H., and R.J.F. analyzed data; S.B.K., B.J.H., and R.J.F. wrote the paper. thirds of this patient population, but the remaining one-third of the population continues to experience seizures (Kwan and Brodie, 2000; Lindsten et al., 2001). Many refractory types of epilepsy result from temporal lobe epilepsy, usually with accompanying mesial temporal lobe sclerosis (Kwan and Brodie, 2000; Perry and 
Duchowny, 2013). Given the complexities of genetic heterogeneity and the inherent difficulties of studying the pathophysiology of epileptogenesis in humans, animal models serve important roles for (1) understanding how recurrent, unprovoked seizures develop (Lemos and Cavalheiro, 1995; Goodman, 1998; Pitkänen et al., 2006); (2) determining whether seizure occurrences remit; and (3) examining the underlying mechanisms of remission.

Remission of recurrent, unprovoked seizures can be achieved in two-thirds of cases by treatment with anticonvulsant medication, surgical resection, and/or nerve/brain electrode stimulation (Krishna et al., 2016; Schachter, 2016). However, there is evidence in human epilepsy of recurrent, unprovoked seizures remitting without any intervention (Sander, 1993; Sander and Shorvon, 1996; Kwan and Sander, 2004; Berg et al., 2006; Callaghan et al., 2007; Luciano and Shorvon, 2007). Elucidating how recurrent, unprovoked seizures develop is critical to the study of epileptogenesis and, in combination with developing a better understanding of how spontaneous seizures remit, may expand our understanding and treatment of epilepsy (Kwan and Sander, 2004).

In the present study, we perform long-term video-electroencephalographic (EEG) recordings in the comparatively seizureresistant C57BL/6J mouse strain following exposure to eight flurothyl-induced generalized seizures (1 seizure/d; Papandrea et al., 2009). Flurothyl, a GABA $A_{A}$ antagonist, is a volatile chemoconvulsant that was previously used to induce seizures in severely depressed patients as an alternative to electroconvulsive shock therapy (Krasowski, 2000; Fink, 2014). There are two primary advantages to the use of flurothyl as a chemoconvulsant. First, flurothyl is rapidly eliminated through the lungs with no detectable metabolism, greatly reducing potential confounds due to residual drug effects (Krantz et al., 1957; Dolenz, 1967; Adler, 1975). Second, rapid drug elimination results in shorter flurothyl-induced seizure durations (e.g., typically 15-60 s, depending on the seizure type expressed) once animals are exposed to room air.

Here, we show that following eight flurothyl-induced seizures C57BL/6J mice develop spontaneous seizures that persist for $\sim 4$ weeks, followed by remission of these spontaneous seizure events. This primary generalized or idiopathic generalized seizure model, producing spontaneous seizures with occurrence remission over time, is distinct from existing spontaneous seizure models and may advance our understanding of the pathophysiological mechanisms underlying epilepsy. This model may also help to understand why seizures can remit without therapeutic intervention.

\section{Materials and Methods}

Animals. Male C57BL/6J mice were used in all experiments [C57 BL/6J, The Jackson Laboratory (http://jaxmice.jax.org/strain/000664. html)]. Mice were purchased and arrived at the Albany Medical College Animal Resource Facility at 6 weeks of age. Mice were allowed complete access to food and water, and were on a standard $12 \mathrm{~h} \mathrm{light/dark}$ cycle with lights on at 7:00 A.M. All experimental protocols were approved by the Institutional Animal Care and Use Committee of Albany Medical College, and were in compliance with the National Institutes of Health Guide for the Care and Use of Laboratory Animals.

\section{${ }^{\dagger}$ Deceased, August 4, 2015.}

The authors declare no competing financial interests.

Correspondence should be addressed to Dr. Russell J. Ferland, Albany Medical College, Department of Neuroscience and Experimental Therapeutics, 47 New Scotland Avenue, MC-136, Albany, NY 12208. E-mail: ferlanr@mail.amc.edu.

DOI:10.1523/JNEUROSCI.3232-14.2016

Copyright $\odot 2016$ the authors $\quad 0270-6474 / 16 / 367486-12 \$ 15.00 / 0$
Surgical procedures for electrode implantation. All surgical procedures were performed when mice were 6 weeks old. Induction of anesthesia was attained with $3 \%$ isoflurane and maintained with $1.5 \%$ isoflurane. Twisted bipolar electrodes (Plastics One) were implanted into the hippocampus, targeting the dentate gyrus [anteroposterior (AP), - 1.6; mediolateral (ML), 0.8; dorsoventral (DV), -1.9; Paxinos and Franklin, 2001]. The placements of hippocampal electrodes were verified following brain sectioning and were found to be on average (mean \pm SEM) at $-1.79 \pm 0.02 \mathrm{AP}, 0.8 \pm 0.03 \mathrm{ML}$, and $1.9 \pm 0.03 \mathrm{DV}$ (Paxinos and Franklin, 2001). Cortical screws were placed over the parietal, frontal, and occipital cortices. The screw in the occipital bone served as a common ground for both the hippocampal and cortical electrodes, with the parietal and frontal screws serving as cortical electrodes. Mice were administered buprenorphine $(0.1 \mathrm{mg} / \mathrm{kg}$, s.c.) for postsurgical pain control. Following surgery, mice were housed individually, with food and water available ad libitum. Mice were allowed to recover for 1 week before flurothyl testing began.

Flurothyl seizure induction. Individual mice (7 weeks old) were exposed to either $10 \%$ or $100 \%$ flurothyl (bis(2,2,2-trifluoroethyl) ether (Sigma-Aldrich)). Flurothyl was administered to mice in a $1.5 \mathrm{~L}$ closed Plexiglas chamber. To achieve a $10 \%$ flurothyl concentration, flurothyl was diluted in $95 \%$ ethanol. We determined that C57BL/6J mice exposed to $10 \%$ flurothyl in $95 \%$ ethanol had low blood alcohol levels of $0.011 \pm$ $0.006 \%$ following $4 \mathrm{~min}$ of exposure. Flurothyl binds at the $\mathrm{GABA}_{\mathrm{A}}$ receptor, where it acts as a noncompetitive antagonist (Krasowski, 2000). Given that a receptor that is bound by a noncompetitive antagonist will no longer be activated by the binding of an agonist, and that ethanol is a positive allosteric modulator of the $\mathrm{GABA}_{\mathrm{A}}$ receptor, ethanol is unlikely to exert a major effect at this receptor in the presence of flurothyl.

Ten percent flurothyl was infused via a syringe pump (Kent Scientific) at a rate of $6 \mathrm{ml} / \mathrm{h}$ onto a gauze pad suspended at the top of the chamber. Since flurothyl is highly volatile, it rapidly vaporizes, leading to inhalation and subsequent seizure expression. The generalized seizure threshold (GST) was defined as the latency from the commencement of the infusion of flurothyl to the occurrence of loss of postural control in the animal. Once the animal lost its posture (i.e., expressed a generalized clonic seizure, Grades 1-2), the chamber was opened to fresh air, resulting in the rapid elimination of flurothyl. Upon seizure resolution, the animal was returned to its home cage until the next day. Mice were given one $10 \%$ flurothyl-induced seizure per day for $8 \mathrm{~d}$ (the flurothyl induction phase) followed by a 4 week (group 1) or 8 week (group 2) rest period during which EEGs were continuously obtained. Animals were retested with a single flurothyl rechallenge following the termination of the long-term EEG recordings. The following four control (ctl) groups were also used: (1) exposure to one $10 \%$ flurothyl-induced seizure, (2) exposure to five $10 \%$ flurothyl-induced seizures, (3) exposure to eight ethanol-only trials (using the average flurothyl exposure time per trial as the amount of ethanol delivered), and (4) exposure to eight flurothylinduced seizures at a concentration of $100 \%$ flurothyl ( $50 \mu$ l of flurothyl at a $100 \%$ concentration was pipetted directly into the chamber and onto a gauze pad). For this last group, flurothyl was delivered as a bolus, instead of being infused, due to the volatile nature of $100 \%$ flurothyl. At the infusion rates required, significant evaporation of the flurothyl within the infusion tubing would occur and result in imprecise delivery of flurothyl. Importantly, this last control group was included as a control for the possible effects of the ethanol diluent on the development of spontaneous seizures expressed after flurothyl induction. After the above exposures, mice were continually recorded $24 \mathrm{~h} / \mathrm{d}$ and $7 \mathrm{~d} /$ week via video-EEG.

Flurothyl-induced seizures were scored on the following graded scale: Grade 1, a loss of posture associated with facial clonus, including chewing and clonus of forelimbs and/or hindlimbs; Grade 2, Grade 1 seizure followed by recovery of the righting reflex and low-intensity bouncing; Grade 3, features of Grade 1 and 2 seizures with recovery of the righting reflex followed by wild running and popcorning; Grade 4, Grade 3 seizure followed by forelimb and/or hindlimb treading; Grade 5, Grade 3/4 seizure followed by bilateral tonic extension of the forelimbs; Grade 6, Grade 5 seizure followed by bilateral tonic extension of the hindlimbs; and Grade 7, Grade 6 seizure followed by immediate death (Samoriski 
and Applegate, 1997). In general terms, Grades 1 and 2 were clonicforebrain seizures comparable to a Grade 5 seizure on the Racine scale for electrical kindling (Racine, 1972), whereas Grades 3-7 were brainstem (tonic) seizures. Importantly, mice recovered their righting reflex before transitioning into Grades 3-7 (Kreindler et al., 1958; Browning and Nelson, 1986). We categorized mice having Grade 3-7 seizures as forebrain $\rightarrow$ brainstem seizures to denote the progression of this type of seizure event.

Experimental procedure for long-term video-EEG monitoring. Mice implanted with electrodes were exposed to one flurothyl-induced seizure $(n=4)$, five flurothyl-induced seizures $(n=8)$, eight ethanol exposures ( $n=4)$, eight flurothyl-induced seizures ( $10 \%$ flurothyl; $n=26)$, eight flurothyl-induced seizures ( $100 \%$ flurothyl; $n=8)$, or had no flurothylinduced seizures (controls; $n=9$ ). Mice were then monitored for spontaneous seizures for up to 8 weeks. For long-term monitoring, mice were housed individually in a custom-made Plexiglas cylindrical cage with dimensions of $24 \mathrm{~cm}$ in diameter and $25 \mathrm{~cm}$ in height. Electrodes were connected through a commutator (Plastics One) to a preamplifier (A-M Systems). The amplified signal was then passed through an amplifier (Model 1800, A-M Systems) where it was further amplified, filtered (between 1 and $500 \mathrm{~Hz}$ ), and digitized at a sampling rate of $1024 \mathrm{~Hz}$ using a notch filter of $60 \mathrm{~Hz}$. Video and EEG recordings were synchronized for analysis of both electrographic and behavioral seizure grading (DataWave Technologies). All mice were recorded continuously $24 \mathrm{~h} / \mathrm{d}, 7$ $\mathrm{d} /$ week.

Seizure detection and data analysis. EEG data files were manually analyzed for electrographic seizures. The electrographic seizures were verified by video, and the behavioral seizure type determined. Some of the early spontaneous electrographic seizures observed had very subtle behavioral manifestations. Spontaneous seizures were defined electrographically as having high-frequency $(>5 \mathrm{~Hz})$ and high-amplitude (more than twice the baseline) rhythmic epileptiform activity with a minimum duration of $10 \mathrm{~s}$. Collected data were further analyzed for (1) seizure frequency, (2) percentage of mice expressing spontaneous seizures, (3) duration of these seizure episodes, and (4) the circadian pattern of the seizure events (day vs night).

Anticonvulsant drug studies. For anticonvulsant drug studies, C57BL/6J mice were administered valproate $[125 \mathrm{mg} / \mathrm{kg}(n=6)$ or 250 $\mathrm{mg} / \mathrm{kg}(n=9)]$ or phenytoin $(20 \mathrm{mg} / \mathrm{kg})$, with additional mice given vehicle injections ( $0.9 \%$ normal saline, $n=10)$. All of these mice received eight induction phase flurothyl-induced seizures and were monitored for spontaneous seizures via continuous long-term video-EEG. Anticonvulsant drugs were administered intraperitoneally, beginning $2 \mathrm{~h}$ following the last seizure on flurothyl trial 8, and then for $24 \mathrm{~d}$. Valproate, phenytoin, or $0.9 \%$ normal saline solution were injected twice daily. Both drug and vehicle solutions were injected in a volume of $0.01 \mathrm{ml} / \mathrm{g}$ body weight. Drug administration was stopped during the last week of the incubation/ recording period, specifically on day 24 . Following this drug washout period of $5 \mathrm{~d}$ (at least seven half-lives; Applegate et al., 1997), mice were given a flurothyl rechallenge. The $5 \mathrm{~d}$ drug washout period permitted the complete elimination of drugs, as the half-life of valproate in mice is $\sim 0.8$ $\mathrm{h}$, and the half-life of phenytoin is $16 \mathrm{~h}$ (Applegate et al., 1997).

Fluoro-Jade B staining. Cell death assays were performed using FluoroJade B staining on brain sections from mice exposed to flurothyl-induced seizures. For this, mice were overdosed with pentobarbital and perfused with PBS followed by $4 \%$ paraformaldehyde in PBS after the following: (1) a single flurothyl seizure; (2) eight flurothyl seizures; (3) eight flurothyl seizures and a $4 \mathrm{~d}$ recording period; (4) eight flurothyl seizures and a 1 week recording period; (5) eight flurothyl seizures and a 2 week recording period; and (6) eight flurothyl seizures and a 4 week recording period. Mice ( $n \geq 6$ per group) were killed $24 \mathrm{~h}$ following a seizure (with the exception of one group of mice exposed to eight flurothyl-induced seizures and killed $2 \mathrm{~h}$ after seizures). These conditions were compared with control mice having no flurothyl exposure. Brains were sectioned in the coronal plane (40-50 $\mu \mathrm{m}$ thickness) and were mounted on gelatinsubbed Superfrost Plus slides (ThermoFisher Scientific). Slides were air dried and immersed in $1 \%$ sodium hydroxide in $80 \%$ ethanol for $5 \mathrm{~min}$. Slides were then immersed in $70 \%$ alcohol followed by a 2 min distilled water wash. Slides were transferred to a solution of freshly made $0.06 \%$ potassium permanganate for $10 \mathrm{~min}$ and then washed in distilled water for $2 \mathrm{~min}$. A final concentration of $0.0004 \%$ Fluoro-Jade B (Histo-Chem) staining solution was freshly prepared in a $0.1 \%$ acetic acid solution from a $0.01 \%$ stock solution of Fluoro-Jade B. This Fluoro-Jade B solution was applied to slides for $20 \mathrm{~min}$ followed by three distilled water washes for 1 min each. Slides were dried, immersed in xylene for $1 \mathrm{~min}$, and coverslipped using DPX plastic mounting media (Sigma-Aldrich). Sections were imaged using an AxioImager Z1 microscope (Carl Zeiss Microimaging) equipped with an AxioCam MRm camera (Carl Zeiss Microimaging), and were processed with AxioVision release 4.5 software (Carl Zeiss Microimaging).

Statistical analyses. Repeated-measures ANOVAs were used for comparisons between flurothyl-exposed and control groups, across days for GST and for spontaneous seizure scoring during the EEG recording period, followed by Newman-Keuls post hoc tests. Pairwise comparisons were made using $t$ tests to determine significance between flurothyl-exposed and control groups within weeks. One-way ANOVA with post hoc tests were used to determine significance between flurothyl exposed and control groups between weeks. One-way ANOVA with post hoc tests were used to determine significance within flurothyl-exposed groups between weeks. One-sample $t$ tests were used to determine significance between the proportion of spontaneous seizures occurring during the day and night. Statistical analyses were performed using Statistica (StatSoft).

\section{Results}

\section{Generalized seizure thresholds over eight flurothyl-induced seizure trials}

Repeated-measures ANOVAs were used to determine whether there were significant differences in GST across the eight flurothyl-induced seizure induction trials with 10\% (Samoriski and Applegate, 1997; Papandrea et al., 2009) or 100\% flurothyl. C57BL/6J mice showed significant differences in GST across the eight seizure trials that plateaued on the fourth seizure trial for mice exposed to $10 \%$ flurothyl $\left(F_{(7,133)}=34.4, p<0.0001\right.$; Fig. $1 A)$. Conversely, no changes in GST were observed across trials in the $100 \%$ flurothyl group receiving $50 \mu \mathrm{l}$ of flurothyl on each trial $\left(F_{(7,49)}=1.6, p=0.15\right.$; Fig. $\left.1 C\right)$. However, changes in GST might have occurred if $100 \%$ flurothyl was infused at a constant rate; however, due to technical issues, we were unable to infuse the required small amounts of flurothyl with our infusion apparatus. As such, direct comparisons between the plots in Figure 1 cannot be made. All seizures observed during the flurothyl-induced seizure trials in the $10 \%$ flurothyl group were behaviorally Grades 1 or 2 (clonic-forebrain seizures). However, $88 \%$ of the flurothylinduced seizures in the $100 \%$ flurothyl group were behaviorally Grades 1 or 2 clonic-forebrain seizures, with the remaining $12 \%$ of the seizures being forebrain $\rightarrow$ brainstem seizures, behaviorally Grades 3-6 (mean, Grade 5). The generalized seizures observed during the flurothyl induction trials were accompanied by electrographic epileptiform activity (Fig. 1B).

\section{Eight flurothyl-induced seizures triggered the emergence of spontaneous seizures with subsequent remission at 1 month} In C57BL/6J mice exposed to eight flurothyl-induced seizures, 95\% of mice developed spontaneous seizures during the 4 week recording period. Spontaneous seizure episodes (SSEs) appeared at the conclusion of the induction phase and continued to occur throughout the following month of recording (Fig. 2). Representative cortical and hippocampal EEGs of a spontaneous seizure episode from a mouse previously exposed to eight flurothylinduced seizures are presented and show an example of the ictal discharges during a clonic-forebrain seizure (Fig. 2). In contrast, no control mice expressed SSEs, indicating that electrode implantation alone had no effect on the expression of spontaneous seizures (Fig. 2). 
Electrographic seizures could be observed beginning on the evening of the last (eighth) flurothyl-induced seizure (day 0) in $~ 50 \%$ of the $\mathrm{C} 57 \mathrm{BL} / 6 \mathrm{~J}$ mice monitored (eventually $\sim 95 \%$ of mice express SSEs). These initial SSEs (average duration, $21 \mathrm{~s}$ ) were not accompanied by any gross behavioral manifestations in the majority of SSEs captured on video, with a few SSEs characterized by myoclonic jerk-like behaviors. On day 1 of the recording period, some of these SSEs progressed and presented with more obvious behavioral manifestations, including clonus of the face and forelimb muscles without loss of posture (average SSE duration, $23 \mathrm{~s}$ ). However, some seizures were less obvious, consisting behaviorally of a brief bout (2-3 s) of clonus of the neck muscles that occurred only at the end of the electrographic seizure. Following the first $2 \mathrm{~d}$ of recording, SSEs became more prolonged having an average seizure duration of $34 \mathrm{~s}$, with the seizures being behaviorally characterized entirely as clonic-forebrain seizures associated with loss of postural control (Grade 1). An acute phase occurred during the first week of the recording period, especially from day 3 to day 7 , where mice experienced the maximum number of spontaneous seizures per day (Figs. 2, 3). After this period, SSEs became gradually more prolonged and occasionally extended up to $60 \mathrm{~s}$ in duration with a prolonged clonic phase. Upon the termination of individual spontaneous seizures, mice experienced varying degrees of postictal depression and immobility. The predominant behavioral seizure phenotype observed throughout the recording period was a Grade 1 or 2 seizure (clonic-forebrain seizure as previously defined (Kreindler et al., 1958; Browning and Nelson, 1986; Samoriski and Applegate, 1997)), but occasionally a mouse had a Grade 3 (running and bouncing seizure) or Grade 4 (running and bouncing followed by a treading seizure) brainstem seizure. Grade 3/4 seizures were clonic seizures that rapidly transitioned into a brainstem seizure phenotype, as previously behaviorally defined (Kreindler et al., 1958; Browning and Nelson, 1986; Samoriski and Applegate, 1997). The electrographic characteristics of a Grade 3-4 forebrain $\rightarrow$ brainstem seizure consisted of high-frequency, high-amplitude epileptiform activity occurring during the Grade 1/2 clonic-forebrain seizure that diminished as the animal transitioned into a Grade 3/4 behavioral brainstem seizure, such that both hippocampal and cortical recordings indicated potential inhibition similar to postictal depression (Fig. 4; the higher-frequency burst at the beginning of the brainstem seizure at the cortical screw electrode was due to the noise of the running-bouncing seizure). Approximately $7 \%$ of the recorded spontaneous seizures were Grade 3 or
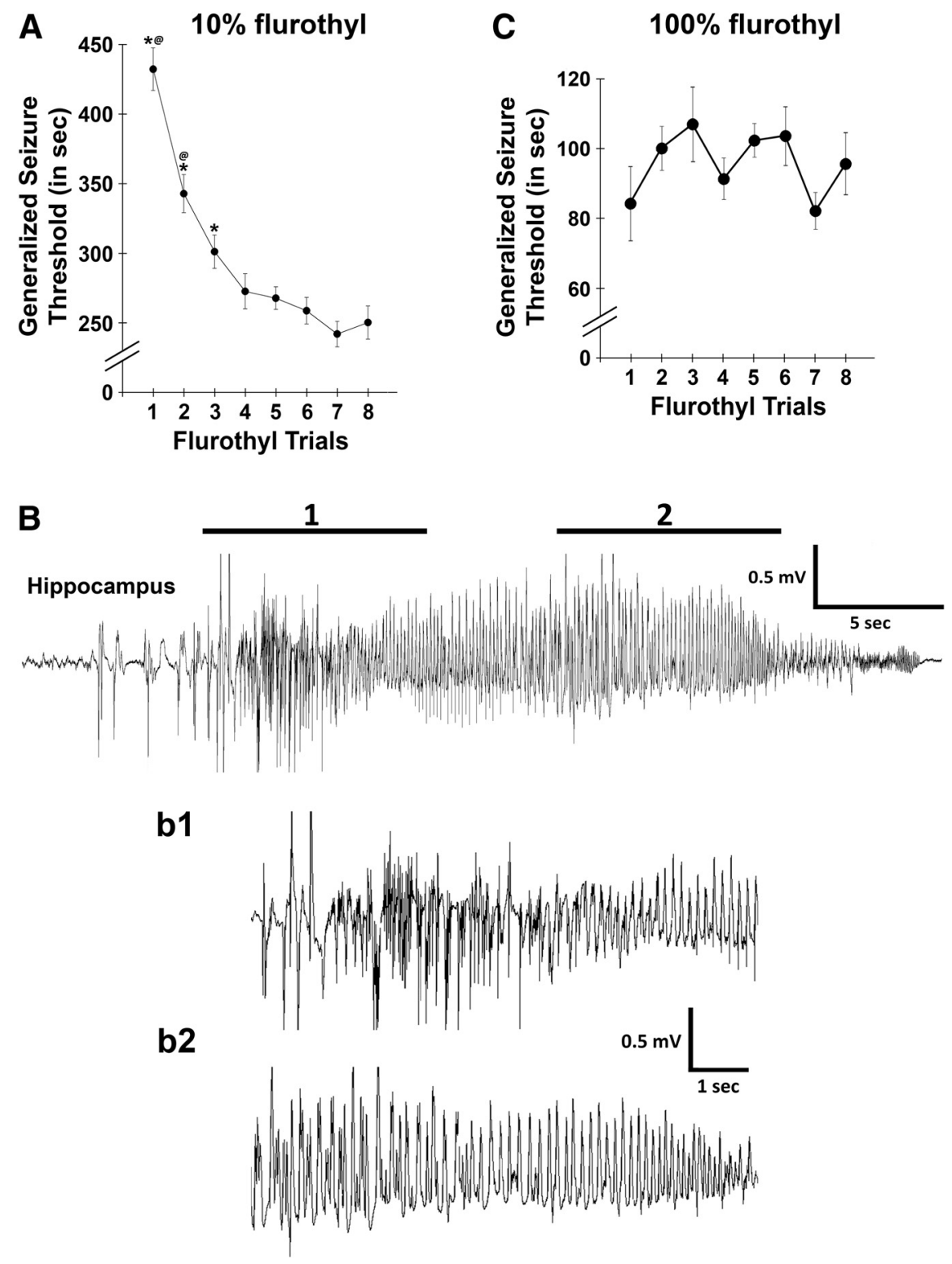

Figure 1. $\quad(57 \mathrm{BL} / 6 \mathrm{~J}$ mice showed decreases in generalized clonic seizure thresholds with repeated flurothyl-induced seizures using $10 \%$ flurothyl. $\boldsymbol{A}$, The latency to a generalized clonic seizure (i.e., GST) on each seizure trial was assessed for (57BL/6J mice by exposure to $10 \%$ flurothyl during eight seizure induction trials $(1 / \mathrm{d})$. There was a significant decrease in GST following repeated clonic seizures $(p<0.0001)$ until plateauing on flurothyl trial 4 . *Significance $(p<0.0009)$ compared with other asterisk groups; ${ }^{@}$ significance $(p<0.009)$ compared with trials $3-8$, as determined by Newman-Keuls post hoc comparisons (trial 3 is also significantly different from trials $6-8, p \leq 0.05)$. $\boldsymbol{B}$, Representative hippocampal local field potential recorded from a (57BL/6J mouse exposed to flurothyl expressing a generalized clonic seizure (Grade 1). $\boldsymbol{B} \boldsymbol{b} 1, \boldsymbol{B} \boldsymbol{b} 2$, Higher-resolution traces are shown for two representative regions of the trace in $\boldsymbol{B}$. C, The latency to a generalized seizure (GST) on each seizure trial was assessed for (57BL/6 J mice by exposure to $100 \%$ flurothyl during eight seizure induction trials (1/d). Plots denote mean \pm SEM.

4 behavioral forebrain $\rightarrow$ brainstem seizures (five forebrain $\rightarrow$ brainstem seizures in week 1 , five forebrain $\rightarrow$ brainstem seizures in week 2 , and one forebrain $\rightarrow$ brainstem seizure in week 3; 33\% of mice had repeat forebrain $\rightarrow$ brainstem seizures). None of the mice were observed having forelimb extension (Grade 5) or hindlimb extension (Grade 6). Moreover, there was no correlation between the length of the clonic seizure and the likelihood of transitioning into a brainstem seizure. Last, we observed no mortality during the $24 \mathrm{~h}, 7 \mathrm{~d}$ /week video-EEG monitoring.

Repeated-measures ANOVA, comparing flurothyl-exposed (10\% flurothyl) and control mice, determined that SSEs were significantly different between groups across the entire recording 
Number of spontaneous seizure events (SSE) following eight $10 \%$ flurothyl-induced seizures and a 26 day recording period

\begin{tabular}{|c|c|c|c|c|c|c|c|c|c|c|c|c|c|c|c|c|c|c|c|c|c|c|c|c|c|c|c|}
\hline ID & ay 1 & $0 \mathrm{ayz}$ & Day & $\mathrm{D}$ & \begin{tabular}{|l|l|}
4 & $\mathrm{Da}$ \\
\end{tabular} & & Day 6 & Day 7 & Day 8 & 8 Day 9 & Day 10 & Day 11 & Day 12 & \begin{tabular}{|l|l|}
2 & Day 13
\end{tabular} & Day 14 & 4 Day 15 & 5 Day 16 & Day 17 & Day 18 & \begin{tabular}{|l|l|}
8 19 \\
\end{tabular} & 9 Day 20 & 0 & 1 Day 22 & \begin{tabular}{|l|l|}
2 & Day \\
\end{tabular} & Day 24 & (1) $\mathrm{Day} 25$ & 5 Day 26 \\
\hline 1 & & 1 & 2 & 2 & 1 & & 1 & 1 & 0 & 1 & 1 & 0 & 0 & 0 & 2 & 0 & 0 & 1 & 3 & 1 & 0 & 0 & 0 & 0 & 0 & 0 & \\
\hline 2 & & 1 & 3 & 3 & & & 0 & 0 & 0 & 0 & 0 & 0 & 3 & 2 & 2 & 0 & 0 & 0 & 0 & 0 & 0 & 0 & 0 & 0 & 0 & 0 & \\
\hline 3 & & 1 & 1 & 3 & & 2 & 3 & 3 & 2 & 0 & 0 & 0 & 2 & 1 & 1 & 0 & 0 & 0 & 0 & 0 & 2 & 0 & 1 & 3 & 1 & 0 & 0 \\
\hline 4 & & 0 & 3 & 1 & 2 & & 1 & 3 & 1 & 1 & 1 & 0 & 0 & 0 & 0 & 0 & 2 & 1 & 0 & 1 & 1 & 1 & 0 & 0 & 1 & 0 & 1 \\
\hline 5 & 0 & 2 & 2 & 3 & & & 3 & 2 & 0 & 0 & 0 & 0 & 0 & 1 & 0 & 0 & 1 & 0 & 1 & 1 & 2 & 0 & 1 & 1 & 2 & 0 & 0 \\
\hline 6 & 1 & 0 & 0 & 2 & & 1 & 1 & 4 & 5 & 2 & 2 & 0 & 0 & 0 & 0 & 0 & 0 & 0 & 0 & 0 & 0 & 0 & 0 & 0 & 1 & 0 & 0 \\
\hline 7 & 0 & 0 & 1 & 0 & & & 1 & 4 & 5 & 1 & 3 & 1 & 1 & 0 & 0 & 0 & 0 & 0 & 0 & 0 & 2 & 2 & 0 & 0 & 0 & 0 & 0 \\
\hline 8 & 1 & 0 & 1 & 1 & 2 & & 3 & 1 & 0 & 0 & 0 & 0 & 0 & 0 & 0 & 1 & $\frac{4}{2}$ & $\frac{0}{2}$ & 0 & 0 & $\frac{2}{0}$ & 0 & 0 & 0 & 0 & 0 & 0 \\
\hline 9 & 2 & 1 & 4 & 3 & & & 1 & 0 & 0 & 2 & 2 & 2 & 1 & 0 & 0 & 0 & 0 & 0 & 3 & 2 & 0 & 0 & 0 & 0 & 0 & 0 & 0 \\
\hline 10 & 1 & 1 & 2 & 2 & & 3 & 2 & 0 & 0 & 0 & 2 & 5 & 0 & 1 & 0 & 0 & 0 & 0 & 0 & 0 & 0 & 2 & 1 & 0 & 0 & 0 & 0 \\
\hline 11 & 0 & 2 & 3 & 4 & 3 & & 4 & 4 & 3 & 3 & 2 & 0 & 0 & 0 & 0 & 0 & 2 & 0 & 0 & 1 & 2 & 0 & 0 & 0 & 0 & 0 & 0 \\
\hline 12 & 0 & 0 & 3 & 2 & $\frac{6}{6}$ & 6 & 4 & 0 & 0 & 1 & 0 & 0 & 1 & 0 & 0 & 0 & 0 & 0 & 0 & 0 & 0 & 0 & 0 & 0 & 0 & 0 & 0 \\
\hline 13 & 1 & 3 & 0 & 2 & & & 5 & 2 & 1 & 3 & 1 & 1 & 0 & 0 & 2 & 2 & & & & & & & & & & & \\
\hline 14 & 0 & 0 & 0 & 0 & & & 3 & 4 & 1 & 0 & 0 & 0 & 1 & 0 & 0 & 0 & 0 & 0 & 0 & 0 & 0 & 0 & 0 & 0 & 0 & 0 & 0 \\
\hline 15 & 2 & 1 & 1 & 1 & 2 & 2 & 6 & 3 & 0 & 0 & 0 & 0 & 3 & 2 & 3 & 0 & 0 & 0 & 0 & 0 & 0 & 0 & 0 & 0 & 0 & 0 & 0 \\
\hline 16 & & & & & & & & & & & & & & & & & & & $\frac{0}{1}$ & 1 & 3 & $\frac{0}{2}$ & 1 & 0 & 0 & 0 & 0 \\
\hline 17 & 2 & 5 & 4 & 2 & & 4 & 2 & 0 & 1 & 1 & 2 & 1 & 0 & 1 & 0 & 0 & 0 & 0 & 0 & 3 & 1 & 0 & 0 & 0 & 0 & 0 & 4 \\
\hline 18 & $\frac{2}{1}$ & 4 & $\frac{4}{2}$ & 2 & & $\frac{4}{1}$ & $\frac{2}{1}$ & 2 & 0 & $\frac{1}{1}$ & 0 & 0 & 0 & 0 & $\frac{5}{2}$ & $\frac{5}{2}$ & 0 & 0 & 0 & 0 & 0 & 0 & 0 & 0 & 0 & 0 & 0 \\
\hline 19 & 0 & 0 & 0 & 0 & 1 & $\overline{0}$ & 0 & 0 & 0 & 0 & 0 & 0 & 0 & 0 & 2 & 1 & 0 & 0 & 0 & 0 & 0 & 1 & 1 & 0 & 0 & 0 & 0 \\
\hline 20 & $\frac{0}{1}$ & 5 & $\frac{5}{3}$ & $\frac{5}{5}$ & & & 0 & 0 & $\frac{0}{0}$ & 0 & 0 & 0 & 0 & $\frac{0}{0}$ & 20 & $\frac{1}{1}$ & 0 & 0 & 0 & 0 & 0 & $\frac{1}{0}$ & 0 & 0 & 0 & 0 & $\frac{0}{0}$ \\
\hline
\end{tabular}

\begin{tabular}{|l|l|l|l|l|l|l|l|l|l|l|l|l|l|l|l|l|l|l|l|l|l|l|l|l|l|l|}
\hline CTL-1 & 0 & 0 & 0 & 0 & 0 & 0 & 0 & 0 & 0 & 0 & 0 & 0 & 0 & 0 & 0 & 0 & 0 & 0 & 0 & 0 & 0 & 0 & 0 & 0 & 0 & 0 \\
\hline CTL-2 & 0 & 0 & 0 & 0 & 0 & 0 & 0 & 0 & 0 & 0 & 0 & 0 & 0 & 0 & 0 & 0 & 0 & 0 & 0 & 0 & 0 & 0 & 0 & 0 & 0 & 0 \\
\hline CTL-3 & 0 & 0 & 0 & 0 & 0 & 0 & 0 & 0 & 0 & 0 & 0 & 0 & 0 & 0 & 0 & 0 & 0 & 0 & 0 & 0 & 0 & 0 & 0 & 0 & 0 & 0 \\
\hline CTL-4 & 0 & 0 & 0 & 0 & 0 & 0 & 0 & 0 & 0 & 0 & 0 & 0 & 0 & 0 & 0 & 0 & 0 & 0 & 0 & 0 & 0 & 0 & 0 & 0 & 0 & 0 \\
\hline CTL-5 & 0 & 0 & 0 & 0 & 0 & 0 & 0 & 0 & 0 & 0 & 0 & 0 & 0 & 0 & 0 & 0 & 0 & 0 & 0 & 0 & 0 & 0 & 0 & 0 & 0 & 0 \\
\hline CTL-6 & 0 & 0 & 0 & 0 & 0 & 0 & 0 & 0 & 0 & 0 & 0 & 0 & 0 & 0 & 0 & 0 & 0 & 0 & 0 & 0 & 0 & 0 & 0 & 0 & 0 & 0 \\
\hline CTL-7 & 0 & 0 & 0 & 0 & 0 & 0 & 0 & 0 & 0 & 0 & 0 & 0 & 0 & 0 & 0 & 0 & 0 & 0 & 0 & 0 & 0 & 0 & 0 & 0 & 0 & 0 \\
\hline CTL-8 & 0 & 0 & 0 & 0 & 0 & 0 & 0 & 0 & 0 & 0 & 0 & 0 & 0 & 0 & 0 & 0 & 0 & 0 & 0 & 0 & 0 & 0 & 0 & 0 & 0 & 0 \\
\hline CTL-9 & 0 & 0 & 0 & 0 & 0 & 0 & 0 & 0 & 0 & 0 & 0 & 0 & 0 & 0 & 0 & 0 & 0 & 0 & 0 & 0 & 0 & 0 & 0 & 0 & 0 & 0 \\
\hline
\end{tabular}

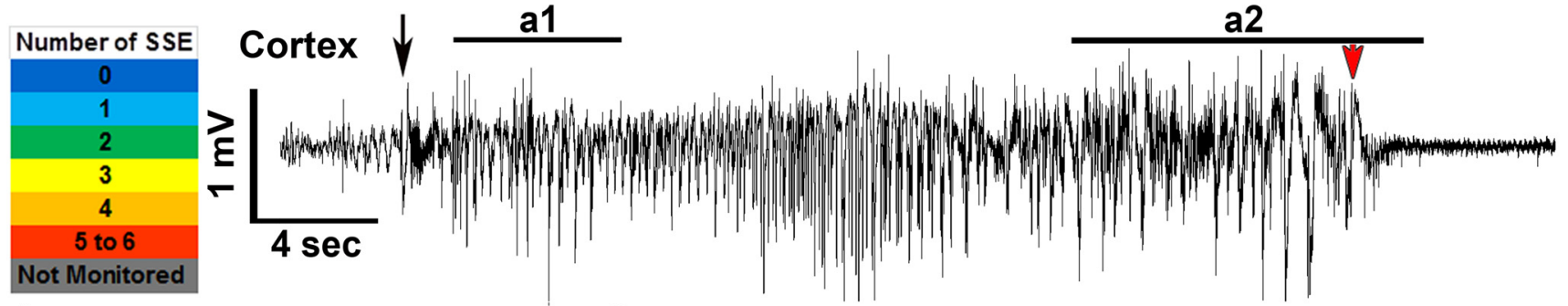

a1

a2

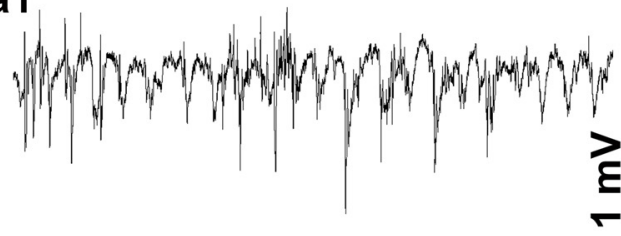

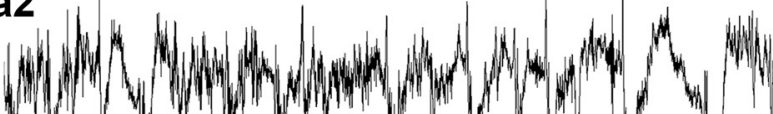

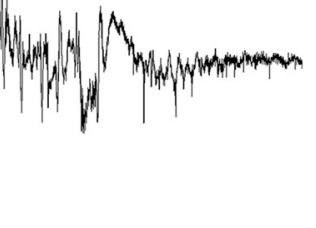

$4 \mathrm{sec}$

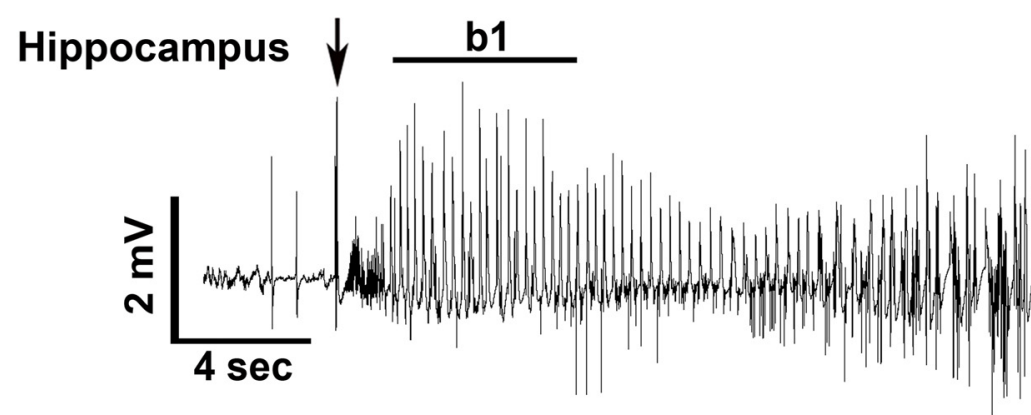

b2

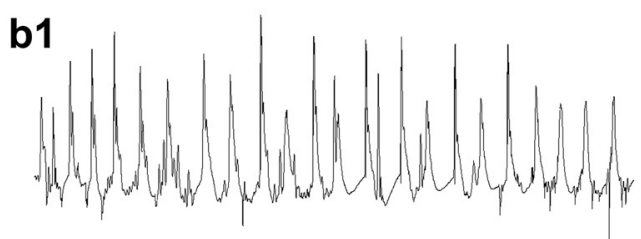

b2
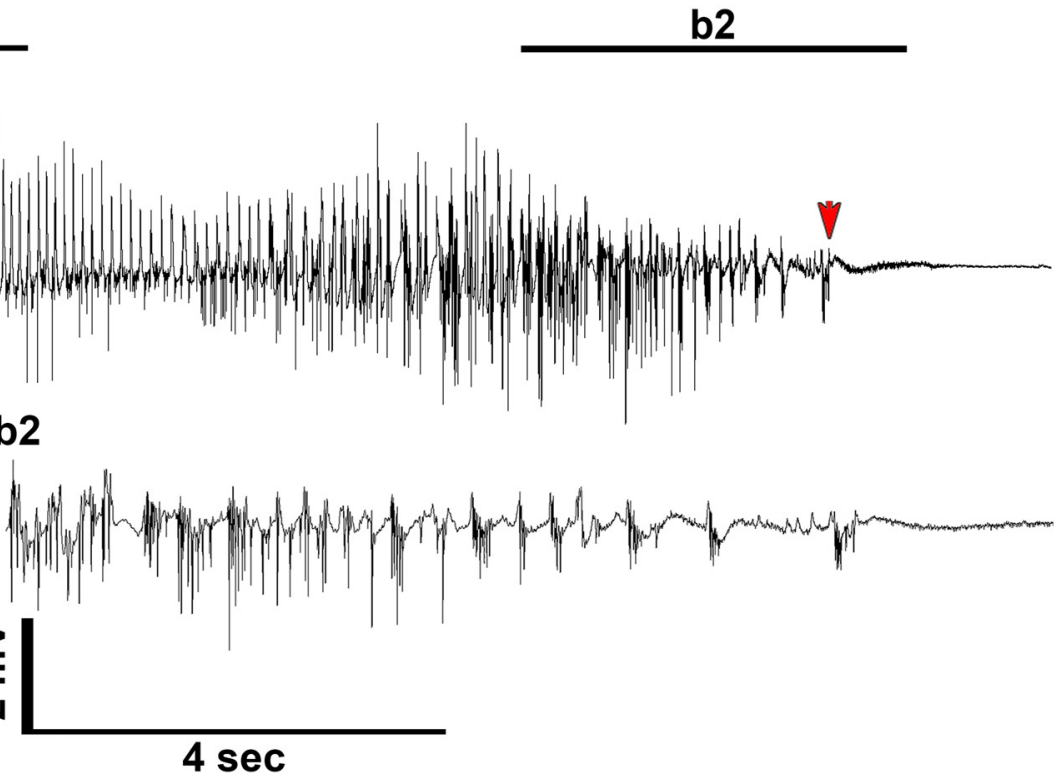

Figure 2. Total number of spontaneous seizure episodes recorded from (57BL/6J mice exposed to eight flurothyl-induced generalized clonic seizures (flurothyl concentration, 10\%). SSEs were detected in $57 \mathrm{BL} / 6 \mathrm{~J}$ mice implanted with cortical and hippocampal electrodes following eight flurothyl-induced seizures. Numbers in the first column indicate animal identifiers. Subsequent columns indicate the day during the recording period following exposure to eight flurothyl-induced seizures (day 0 is the last day of flurothyl exposure) and a 4 week (Figure legend continues.) 

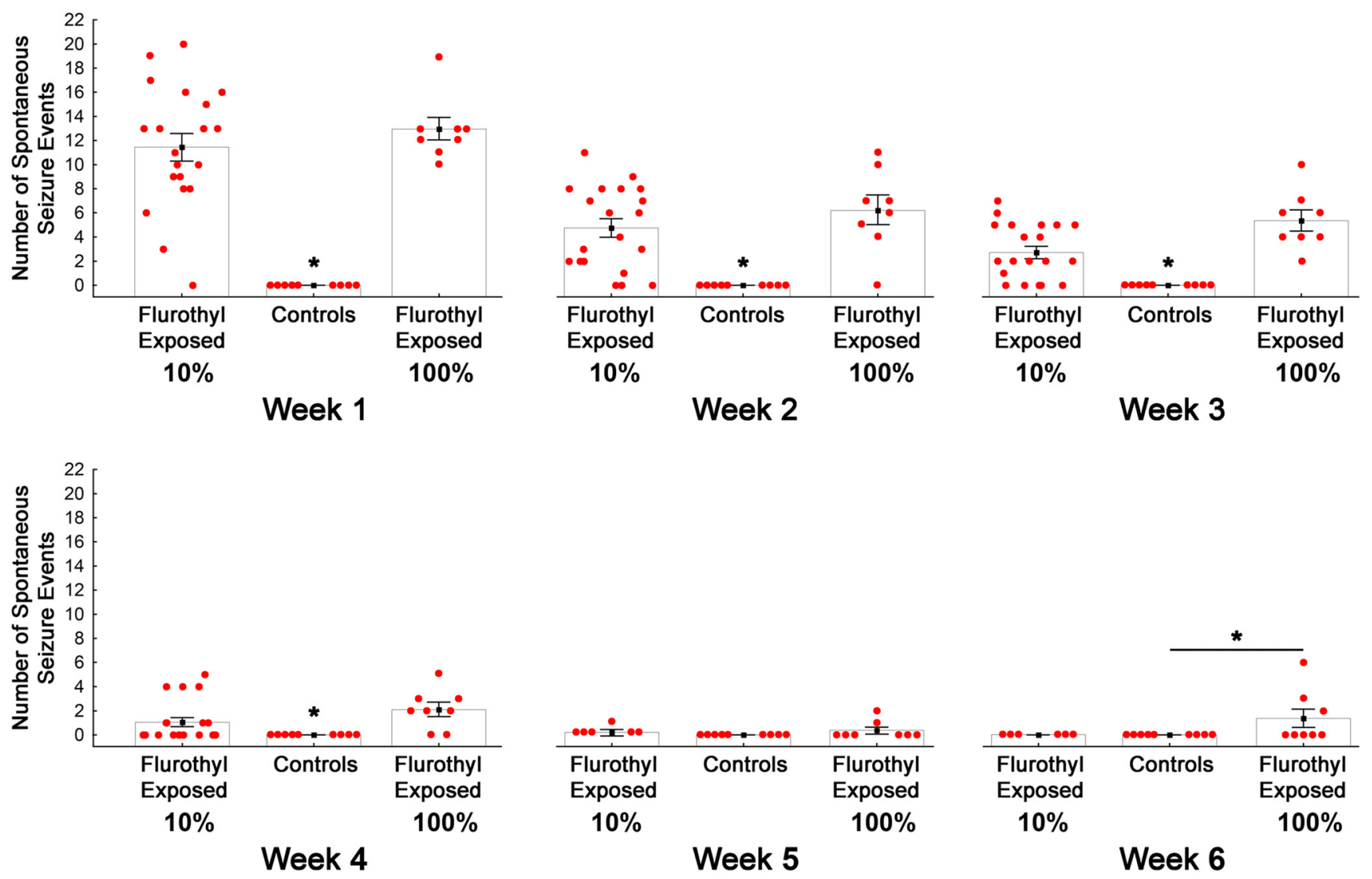

Figure 3. Average number of spontaneous seizures during a 6 week long-term video-EEG recording period in $557 \mathrm{BL} / 6 \mathrm{~J}$ mice previously exposed to eight repeated flurothyl-induced generalized clonic seizures (flurothyl concentrations, 10\% and 100\%). Total number of SSEs detected in (57BL/6J mice that were exposed to either 10\% or 100\% flurothyl, grouped according to weeks. Significant differences exist between flurothyl-exposed and control mice $(p<0.0001)$; days across the recording period $(p<0.0001)$, and for the interaction of days and flurothyl exposure $(p<0.0001)$. Moreover, significant differences (*) between flurothyl-exposed and control mice were found using a within-weeks comparison (week 1, $p<0.004$; week 2, $p<0.0005$; week $3, p<$ 0.003 ; week $4, p<0.02$; week $6, p<0.002$ ). The $x$-axis represents the duration of the recording period by weeks, whereas the $y$-axis represents the mean number of seizures expressed (light gray bar; black filled circle) and the SEM. The number of seizures each individual mouse expressed is represented by the filled red circles.

period $\left(F_{(1,27)}=54.9, p<0.0001\right.$; Fig. 2). Similarly, SSEs were significantly different across the days of the recording period $\left(F_{(27,729)}=3.4, p<0.0001\right)$. The interaction between days of the recording period with flurothyl exposure was also determined to be significantly different $\left(F_{(27,729)}=3.6, p<0.0001\right.$; Fig. 2$)$. We binned the total number of SSEs by weeks and then compared flurothyl-exposed and control groups. One-factor ANOVA demonstrated that these differences were significant between the flurothyl-exposed and control groups $\left(F_{(1,110)}=62.8, p<\right.$ $0.0001)$ and by week $\left(F_{(3,110)}=12.4, p<0.0001\right.$; Fig. 3). Comparisons were made within the weeks and between the flurothylexposed and control groups using $t$ tests, which were determined to be significant (week 1: Sz vs ctl, $t_{(19)}=10.0, p<0.000001$; week 2 : Sz vs ctl, $t_{(19)}=6.2, p<0.00001$; week 3: Sz vs ctl, $t_{(19)}=5.5$, $p<0.0001$; week 4 : Sz vs ctl, $t_{(18)}=2.8, p<0.02$; Fig. 3$)$. These results indicated that spontaneous seizures that were significantly

(Figure legend continued.) recording period. Each individual box is color coded according to the legend. Each of the numbers in these boxes indicates the total number of seizures per day. C57BL/6J mice not given flurothyl-induced seizures (CTL1-9) do not express spontaneous seizures during the recording period. Also presented are low- and high-resolution representative EEGs (local field potentials) from the cerebral cortex (a1 and a2; paired low and high resolution traces) and hippocampus (b1 and b2; paired low and high resolution traces) recorded from a C57BL/6J mouse having a spontaneous generalized clonic seizure (Grade 1). Black arrows denote the beginning of the seizure, and red arrowheads indicate the end of the seizure. different from controls continued to occur during the initial 4 week recording period in mice exposed to $10 \%$ flurothyl. Moreover, comparisons between the percentages of $10 \%$ flurothylexposed mice having spontaneous seizures and controls again demonstrated significant differences within weeks (Fisher exact tests: week $1, p<0.00001$; week $2, p<0.00001$; week $3, p<$ 0.0003 ; week $4, p<0.03$; Fig. 5). Although the number of SSEs dropped over the recording period, the average duration of the SSEs across the 4 weeks was not significantly different (Fig. 6).

To further explore the remission of spontaneous seizures, we exposed another group of animals to flurothyl seizures, as described above, followed by an 8 week video-EEG recording period (Fig. 7). Similar to the situation in the group recorded for 1 month, seizures occurred almost immediately following the last flurothyl-induced seizure and persisted for up to 1 month, with the number of SSEs declining over time. During the second month of recording, no SSEs were observed, indicating that the SSEs had remitted (Fig. 7).

To ascertain whether the appearance of spontaneous seizures depended on the number of flurothyl-induced seizures, additional groups were given either a single flurothyl-induced seizure or five flurothyl-induced seizures, and were then followed by long-term video-EEG recordings. Five seizure trials were chosen, since it had previously been demonstrated that five seizures are required to establish the lowest and permanent generalized seizure threshold for the animals (Samoriski and Applegate, 1997). 


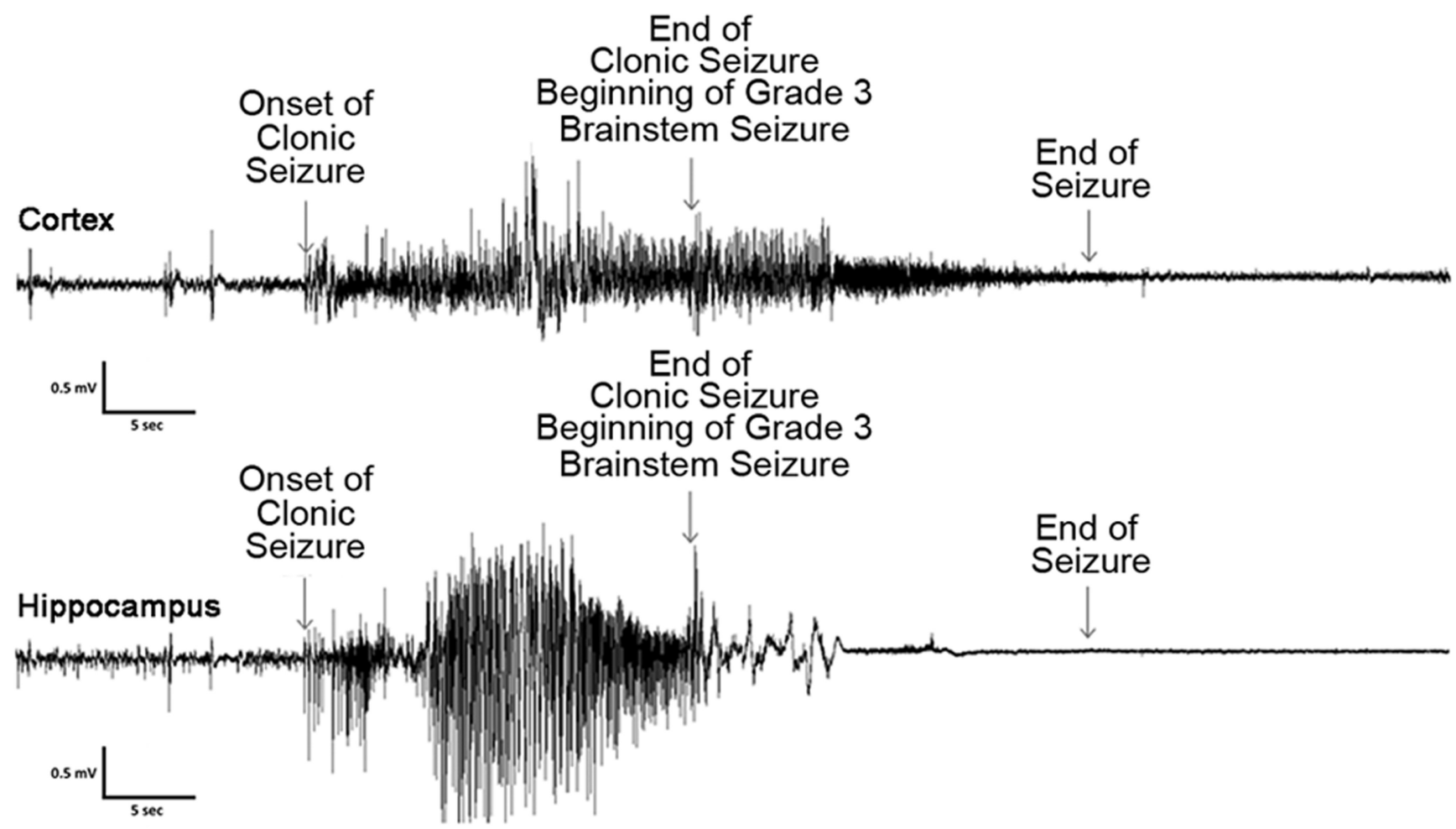

Figure 4. EEG recording from a C57BL/6J mouse having a forebrain $\rightarrow$ brainstem seizure. Both hippocampal and cortical recordings indicate potential cortical/hippocampal inhibition during the behavioral brainstem seizure component of a forebrain $\rightarrow$ brainstem seizure. Top trace represents the cortical EEG recording during the ictal event, whereas the lower trace represents the hippocampal recording. Arrows indicate the onset of the clonic-forebrain generalized seizure and the end of the forebrain seizure. The end of the forebrain seizure is the beginning of the brainstem seizure (which usually begins with running-bouncing seizures, hence the noise following the beginning of the behavioral brainstem seizure), and the next arrow indicates the end of the behavioral brainstem seizure.

One flurothyl-induced seizure did not elicit any spontaneous seizures (data not shown). Five flurothyl-induced seizures resulted in $28 \%$ of the mice developing spontaneous seizures. This suggests that the frequency of SSEs was directly dependent on the number of flurothyl-induced seizures. Also, of the two mice that had SSEs, one animal took $8 \mathrm{~d}$ to develop seizures, and the other developed seizures $2 \mathrm{~d}$ into the EEG recording period (data not shown). This suggested that the time to development of SSEs was also dependent on the number of flurothyl-induced seizures.

Interestingly, we performed a 4 week long-term video-EEG monitoring pilot study in DBA/2J mice exposed to eight flurothyl-induced seizures. Although this strain had fewer total SSEs across the 4 week recording period, the number of spontaneous seizures remained more consistent over the course of 4 weeks (ranging between two and five seizures each week), with DBA/2J mice tending to have more clustered seizures (S.B. Kadiyala and R.J. Ferland, unpublished observations). In addition, the percentage of DBA/2J mice having SSEs did not decrease across the recording period as it did in C57BL/6J mice (S.B. Kadiyala and R.J. Ferland, unpublished observations). This suggested that the background strain may contribute to whether the SSEs continued or remitted.

To confirm that flurothyl, rather than ethanol, was responsible for SSEs (Chopde et al., 1977; Kosobud and Crabbe, 1986), mice were given eight exposures to $95 \%$ ethanol (with no flurothyl) or were given eight seizures using 100\% flurothyl (with no ethanol). Eight exposures to 95\% ethanol did not elicit any SSEs, as assessed with long-term video-EEG recordings (data not shown). However, eight exposures of $100 \%$ flurothyl showed spontaneous seizure expression profiles similar to the $10 \%$ fluro- thyl group, regardless of the mode of administration (Figs. 2, 8). SSEs were significantly different between flurothyl-exposed (100\% flurothyl) and control groups across the entire recording period $\left(F_{(1,15)}=210.7, p<0.0001\right.$; Fig. 8). Similarly, SSEs were significantly different across the days of the recording period for both the $100 \%$ flurothyl-exposed and control groups $\left(F_{(53,795)}=\right.$ $7.4, p<0.0001)$. The interaction between days of the recording period with $100 \%$ flurothyl exposure was also determined to be significantly different $\left(F_{(53,795)}=8.2, p<0.0001\right.$; Fig. 8). Binning total SSEs by weeks and comparing 100\% flurothyl-exposed and control groups demonstrated significant differences between groups $\left(F_{(1,120)}=214.06, p<0.0001\right)$ and weeks $\left(F_{(7,120)}=36.3\right.$, $p<0.0001$; Fig. 3). Direct comparisons of $100 \%$ flurothylexposed and control groups found significance for all week bins, except week 5 (week 1: Sz vs ctl, $t_{(4)}=6.3, p<0.004$; week 2: Sz vs ctl, $t_{(6)}=6.9, p<0.0005$; week 3: Sz vs ctl, $t_{(6)}=4.9, p<0.003$; week 4: Sz vs ctl, $t_{(6)}=4.3, p<0.006$; week 5: $S z$ vs ctl, $t_{(6)}=1.4$, ns; week 6: Sz vs ctl, $t_{(6)}=5.3, p<0.002$; week 7: Sz vs ctl, $t_{(6)}=$ 4.3, $p<0.006$; Fig. 3). Moreover, comparisons between the percentage of $100 \%$ flurothyl-exposed mice having spontaneous seizures and controls also demonstrated significant differences within weeks (Fisher exact tests: week $1, p<0.00001$; week 2, $p<$ 0.00001 ; week 3, $p<0.00001$; week $4, p<00001$; week 5, $p=\mathrm{NS}$; week 6, $p<0.0005$; week 7, $p<0.0005$; Fig. 5). Although individual week differences were not observed via post hoc tests for the average duration of the SSEs across the 7 week recording period, there was a significant overall effect $\left(F_{(5,219)}=5.5, p<0.00001\right.$; Fig. 6 ). Last, $\sim 12 \%$ of the SSEs in mice exposed to $100 \%$ flurothyl were Grade 3 behavioral brainstem seizures (nine brainstem seizures in week 1 , nine brainstem seizures in week 2 , five brainstem 

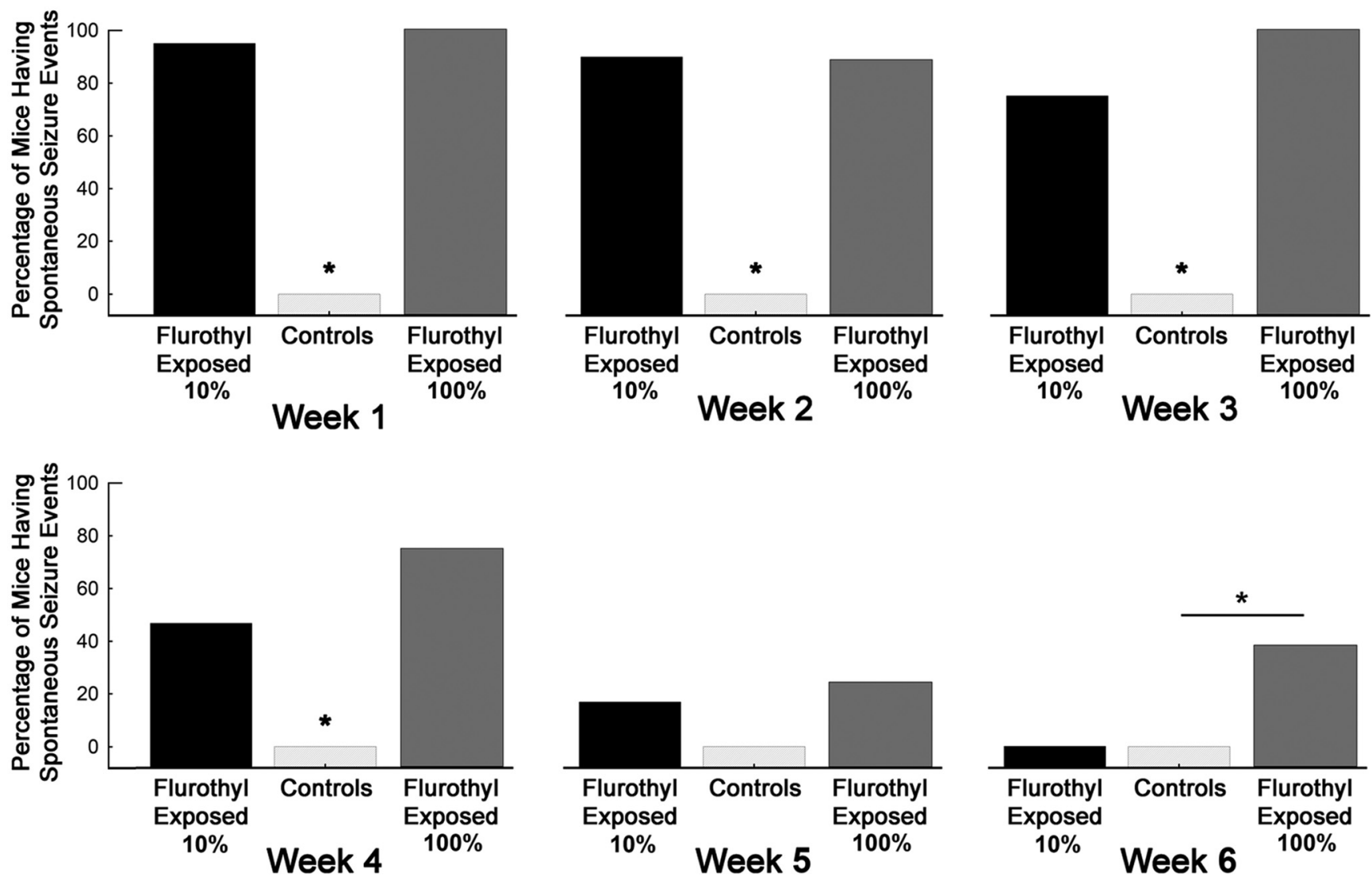

Figure 5. Percentage of $\mathrm{C57BL} / 6 \mathrm{~J}$ mice expressing spontaneous seizures during the long-term video-EEG recording period in mice previously exposed to eight repeated flurothyl-induced generalized seizures (flurothyl concentrations, $10 \%$ and 100\%). Comparisons were made within weeks using Fisher exact tests for the percentage of flurothyl-exposed and control mice having spontaneous seizures. Significant differences ( $\left.{ }^{*}\right)$ were found between flurothyl-exposed and control mice for week $1(p<0.00001)$, week $2(p<0.00001)$, week $3(p<0.0003)$, week $4(p<0.03)$, and week $6(p<0.0005)$. The $x$-axis represents the duration of the recording period by weeks, whereas the $y$-axis represents the percentage of mice having SSES.

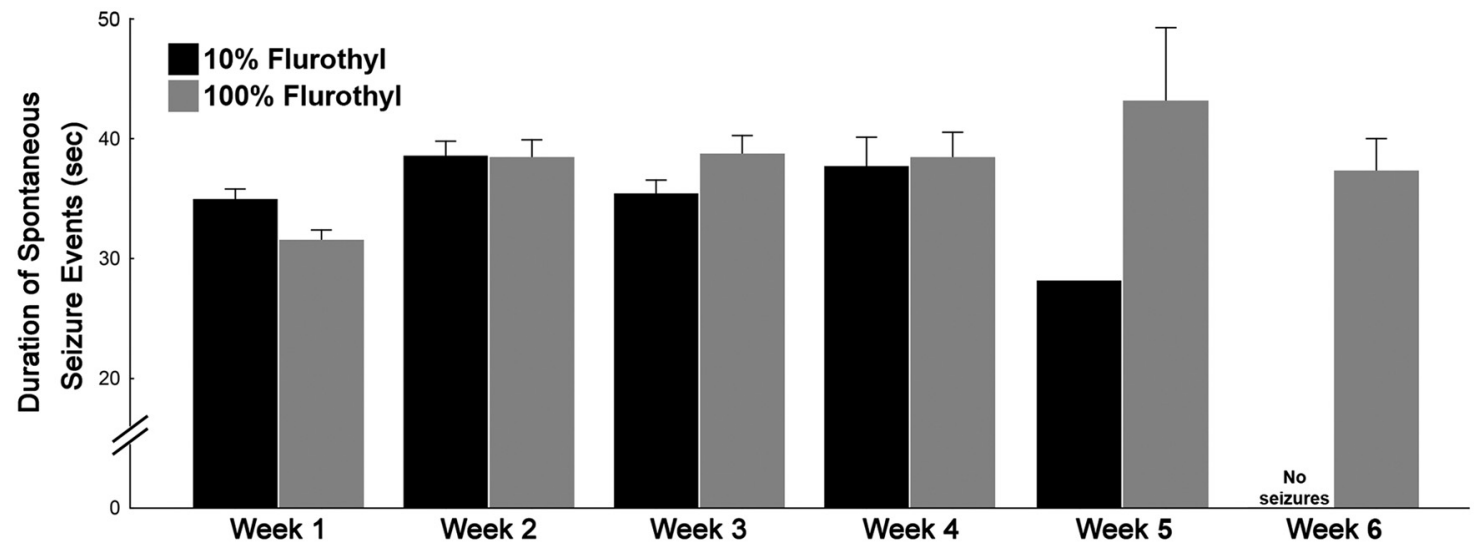

Figure 6. Average duration of spontaneous seizure episodes during the long-term video-EEG recording period in mice previously exposed to eight repeated flurothyl-induced generalized seizures (flurothyl concentrations, $10 \%$ and $100 \%$ ). Average seizure durations were determined from the total number of SSEs detected from C57BL/6J micethat were exposed to either $10 \%$ or $100 \%$ flurothyl. The duration of these seizure events was not significantly different following Newman-Keuls post hoc comparisons across the recording period when binned according to weeks. Plot denotes mean \pm SEM.

seizures in week 3, three brainstem seizures in week 4, and one brainstem seizure in weeks 5 and 6; every mouse examined had repeat brainstem seizures). No mortality was observed during the video-EEG monitoring in the group of mice exposed to $100 \%$ flurothyl-induced seizures.

Examination of the circadian patterns of SSEs in C57BL/6J mice showed that a majority of the seizures were occurring during the day (during the normal sleep cycle for the animals; Fig. 9) with both $10 \%$ and $100 \%$ flurothyl. Significant differences in the number of SSEs existed between day and night during most weeks of the recording period, as determined by one-sample $t$ tests between proportions (weeks $1-6, p<0.05$ ) for both $10 \%$ and $100 \%$ flurothyl-induced seizures (Fig. 9).

No evidence of cell death in brains of mice exposed to repeated flurothyl-induced seizures

In contrast to rodents undergoing status epilepticus and subsequent cell death (Shibley and Smith, 2002; Borges et al., 

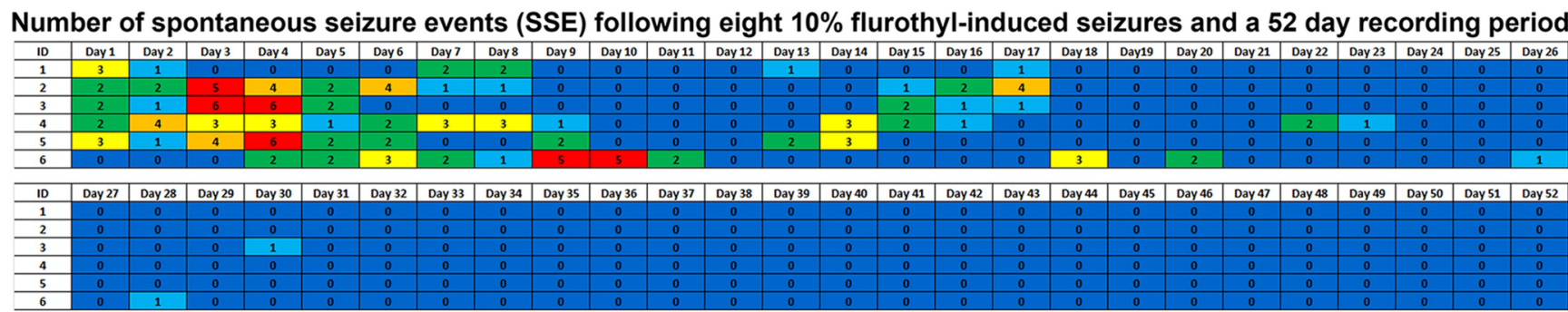

Number of SSE

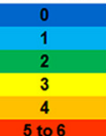

Figure 7. Total number of spontaneous seizure episodes recorded from C57BL/6J mice exposed to eight flurothyl-induced (flurothyl concentration, 10\%) generalized clonic seizures (EEGs were recorded for 8 weeks). SSEs were detected in C57BL/6J mice implanted with cortical and hippocampal electrodes following eight flurothyl-induced seizures (flurothyl concentration, 10\%) over an 8 week recording period. Numbers in the first column indicate animal identifiers. Subsequent columns indicate the day during the recording period following exposure to eight flurothyl-induced seizures (day 0 is the last day of flurothyl exposure). Each individual box is color coded according to the legend. Each of the numbers in these boxes indicates the total number of seizures per day.

Number of spontaneous seizure events (SSE) following eight $100 \%$ flurothyl-induced seizures and a 62 day recording period
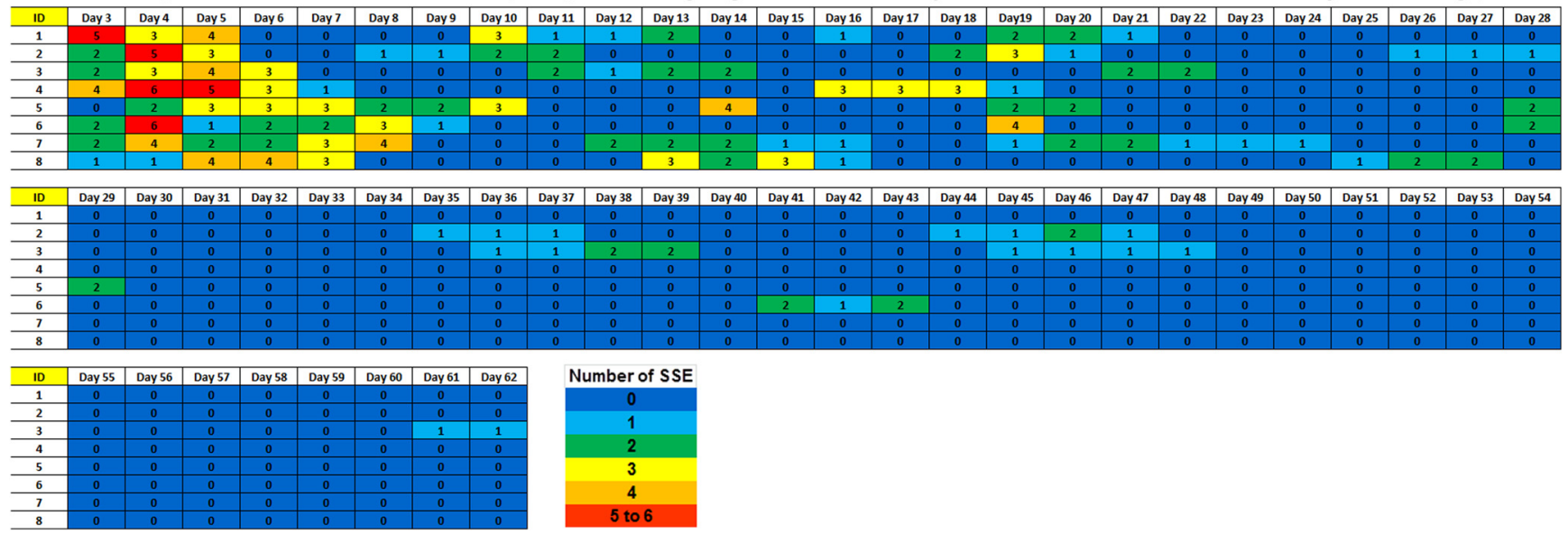

Figure 8. Total number of spontaneous seizure episodes recorded from C57BL/6J mice exposed to eight flurothyl-induced generalized seizures (100\% flurothyl; EEGs were recorded for 8 weeks). SSEs were detected in (57BL/6J mice implanted with cortical and hippocampal electrodes following eight flurothyl-induced seizures (flurothyl concentration, 100\%) over an 8 week recording period. Numbers in the first column indicate animal identifiers. Subsequent columns indicate the day during the recording period following exposure to eight flurothyl-induced seizures (day 0 is the last day of flurothyl exposure). Each individual box is color coded according to the legend. Each of the numbers in these boxes indicates the total number of seizures per day.

2003), mice exposed to repeated flurothyl-induced seizures had no evidence of cell death as assessed by Fluoro-Jade B staining (cell death was observed following pilocarpineinduced status epilepticus and served as a positive control for our Fluoro-Jade B staining). No differences in cell death were observed during the flurothyl induction period (flurothylexposed mice following induction trial 1 or eight induction trials with either a 2 or 24 h kill time post-seizure) or during the recording period ( $4 \mathrm{~d}$ after flurothyl induction, when there were significant numbers of spontaneous seizures, and at the end of the first, second, and fourth weeks of the recording period) compared with control mice (data not shown). These results suggested that the appearance of spontaneous seizures, and their remission, in C57BL/6J mice was not associated with cell death from the flurothyl induction seizures or subsequent spontaneous seizures.

Effect of anticonvulsant drug treatment on spontaneous seizure expression

C57BL/6J mice were exposed to the $10 \%$ flurothyl induction paradigm following the implantation of electrodes and monitored for SSEs for the duration of a 4 week recording (incubation) period. Following the eighth seizure, mice were administered valproate intraperitoneally at two different doses $(125$ or $250 \mathrm{mg} / \mathrm{kg}$, twice daily) or received phenytoin intraperitoneally at a dosage of $20 \mathrm{mg} / \mathrm{kg}$ twice daily or vehicle ( $0.9 \%$ normal saline) intraperitoneally, twice daily. Whereas the saline-injected control mice, the group of mice treated with a $125 \mathrm{mg} / \mathrm{kg}$ dose of valproate, and phenytoin-treated mice expressed spontaneous seizures, the mice treated with a $250 \mathrm{mg} / \mathrm{kg}$ dose of valproate did not develop spontaneous seizures (Fig. 10; $F_{(2,22)}=4.7$, $p<0.03$; Newman-Keuls post hoc tests demonstrated significance at $p<0.03$ between the control group and the group treated with $250 \mathrm{mg} / \mathrm{kg}$ valproate; no significant differences were found among the control, $125 \mathrm{mg} / \mathrm{kg}$ valproate, or phenytoin groups). While the $125 \mathrm{mg} / \mathrm{kg}$ dose of valproate did not block SSEs, it did lower the frequency of spontaneous seizures during the incubation/recording period compared with controls that received saline.

Whereas only clonic-forebrain seizures were observed during the eight seizure flurothyl induction phase with 10\% flurothyl, $95 \%$ of 

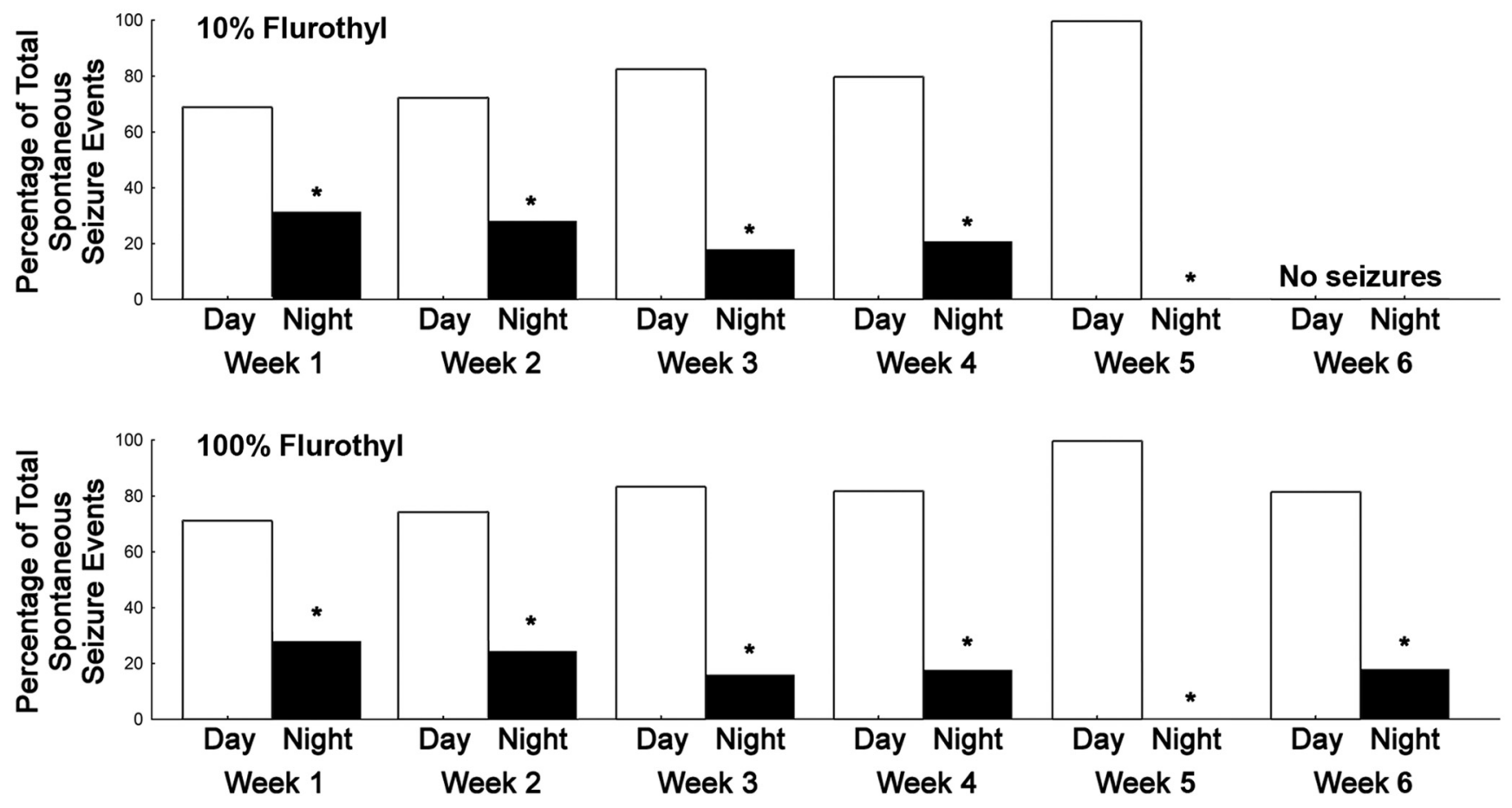

Figure 9. Circadian pattern of seizure distribution in C57BL/6J mice during the long-term video-EEG recording period in mice previously exposed to eight repeated flurothyl-induced generalized seizures (flurothyl concentrations, $10 \%$ and 100\%). The total number of SSEs detected in (57BL/6J mice were grouped by weeks and were further categorized into whether the seizures occurred during the day [lights on (inactive period of mice), 7:00 A.M. to 7:00 P.M.] or night [lights off (active period of mice), 7:00 P.M. to 7:00 A.M.]. Spontaneous seizures occurred significantly more during the lights-on period than in the dark period ( ${ }^{*} p<0.05$ ). The $x$-axis represents the duration of the recording period by weeks, whereas the $y$-axis represents the percentage of total SSEs that occurred during either day (white bars, lights on) or night (black bars, lights off).

these mice experienced a clonic-forebrain seizure that transitioned into a brainstem seizure (forebrain $\rightarrow$ brainstem seizure) upon flurothyl rechallenge after a $28 \mathrm{~d}$ rest period (the time during which the EEGs were recorded). Since C57BL/6J mice that experienced SSEs during the incubation period expressed clonic-forebrain seizures that transitioned into brainstem seizures (a forebrain $\rightarrow$ brainstem seizure) following the conclusion of the 4 week recording period and flurothyl re-exposure, it was conceivable that spontaneous seizures were responsible for the evolution of more complex forebrain $\rightarrow$ brainstem seizures. Following the last anticonvulsant injection, mice went through a drug washout period and a final flurothyl rechallenge. Mice having 8 flurothyl-induced seizures and receiving saline injections during the recording/ incubation period continued to have SSEs. Three of 10 flurothyl-exposed control mice did not express spontaneous seizures, of which two mice had a forebrain seizure on flurothyl rechallenge. These results may indicate that in the absence of spontaneous seizures there was little reorganization; consequently, such mice expressed clonic-forebrain seizures at retest. However, valproate, when administered at a dose of $250 \mathrm{mg} / \mathrm{kg}$, was able to prevent the development of SSEs, and at the completion of the incubation/recording period the flurothyl rechallenge resulted in the blocking of the expression of forebrain $\rightarrow$ brain-

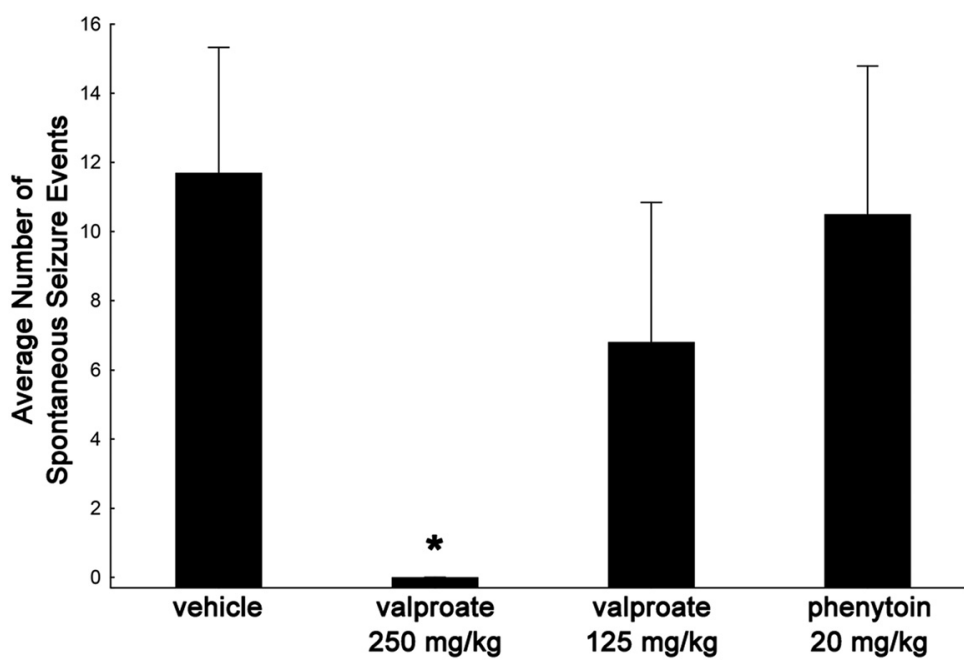

Figure 10. Effect of the anticonvulsant drugs valproate and phenytoin on spontaneous seizure expression during the incubation/recording period in C57BL/6J mice exposed to flurothyl. C57BL/6J mice were exposed to the $8 \mathrm{~d}$ induction paradigm (10\% flurothyl) following the implantation of electrodes and monitored for spontaneous seizures for the duration of the incubation period. Following the eighth seizure (induction trial 8), mice were administered valproate (125 or $250 \mathrm{mg} / \mathrm{kg}$ ), phenytoin (20 $\mathrm{mg} / \mathrm{kg})$, or vehicle $(0.9 \%$ normal saline) twice daily. Vehicle-injected control mice, the group treated with a valproate dose of 125 $\mathrm{mg} / \mathrm{kg}$, and phenytoin-treated mice expressed spontaneous seizures. In contrast, mice receiving $250 \mathrm{mg} / \mathrm{kg}$ valproate did not develop spontaneous seizures $\left({ }^{*} p<0.03\right.$ vs control). Plot denotes mean \pm SEM.

stem seizures in eight of nine mice. This suggested that the spontaneous seizures may have contributed to the evolution of the forebrain $\rightarrow$ brainstem seizure phenotype in C57BL/6J mice. This hypothesis was further supported by data from the groups treated with a $125 \mathrm{mg} / \mathrm{kg}$ dose of valproate and with phenytoin, where mice continued to have SSEs, all of which developed forebrain $\rightarrow$ brainstem seizures on flurothyl rechallenge. 


\section{Discussion}

Our study has shown that eight flurothyl-induced generalized seizures in mice, without status epilepticus or traumatic brain injury, resulted in the expression of spontaneous and recurrent seizures that lasted for up to 1 month, followed by spontaneous seizure remission. Spontaneous seizures tended to occur more during the sleep phase of the mouse circadian cycle, paralleling observations in humans whereby unprovoked seizures tend to occur more often during sleep in many types of epilepsies (Foldvary-Schaefer and GriggDamberger, 2009). We found that $\sim 7-12 \%$ of the spontaneous seizures observed during the recording period could be characterized behaviorally as clonic seizures that transitioned into brainstem seizures. The reduction of forebrain ictal discharge in the EEGs of mice showing behavioral brainstem seizures suggested a forebrain depression during brainstem seizure expression. Last, valproate was able to block the expression of the spontaneous seizures, whereas phenytoin had little effect, indicating that the spontaneous seizures were not blocked by all anticonvulsant drugs. Overall, the present study indicates that this repeated flurothyl paradigm provides a new model of epileptogenesis for understanding mechanisms underlying the development of spontaneous seizures and why spontaneous seizures can remit with time.

Following the induction of status epilepticus in rat models of chemically induced epileptogenesis, there is a latent period during which no seizure activity is observed that is then followed by the appearance of recurrent spontaneous seizures (Leite et al., 1990; Cavalheiro et al., 1991; Lemos and Cavalheiro, 1995). Interestingly, there was no latent period with repeated flurothyl-induced epileptogenesis. This lack of a latent period is unlikely due to residual systemic flurothyl, or its metabolites, since previous pharmacokinetic studies of flurothyl have demonstrated that the majority of the flurothyl that is administered (at a concentration of 100\%) is eliminated in <3 min (Dolenz, 1967; Adler, 1975). Moreover, flurothyl is eliminated from the lungs unchanged with no active metabolites (Adler et al., 1967; Gallagher, 1969; Adler, 1975). Last, studies by Gallagher (1969) demonstrated that repeated flurothyl exposure in rodents did not alter the brain levels of flurothyl, indicating that flurothyl was not lingering in the brain. Given these pharmacokinetic parameters, it is very unlikely that the immediacy of the spontaneous seizures is a result of residual flurothyl. It is intriguing to speculate as to why the animals develop spontaneous seizures without an obvious latent period. Interestingly, a recent report (Mazzuferi et al., 2012) using a mouse model of pilocarpine-induced status epilepticus showed that spontaneous seizures developed within 24-48 h. These results, together with our data, suggest the possibility that mice exposed to repeated flurothyl-induced seizures undergo reorganizational processes leading to spontaneous seizure development during the induction phase of this model. This possibility is in part supported by the fact that five flurothyl-induced seizures can result in a small percentage of mice having spontaneous seizures, and that these spontaneous seizures do not immediately follow the last flurothyl-induced seizure. It also indicates that, in mice, the process underlying the development of spontaneous seizures could be accelerated or distinct, compared with rat models, implicating species differences.

Although flurothyl-induced generalized seizures in the repeated flurothyl model are mild compared with chemical-induced status epilepticus, spontaneous seizures begin to evolve at the conclusion of the flurothyl induction period with robust expression of spontaneous seizures occurring during the first week of the recording period. This indicates distinct differences in seizure progression processes between these models. However, the possibility of an ongoing epi- leptogenic process beginning after the first flurothyl-induced seizure cannot be ruled out, even though one flurothyl-induced seizure is unable to produce SSEs. This is because there is a significant reduction in generalized seizure thresholds over the induction trials. In combination, seizure-induced changes in gene expression and the ensuing compensatory mechanisms that restore homeostasis are likely involved in epileptogenesis and its resolution. These adaptations, along with permanent alterations in neuronal networks, are required for the appearance of spontaneous seizures. Therefore, a single seizure is not sufficient to induce spontaneous seizures, as the above-mentioned changes probably revert to a normal state. But with repeated induced seizures, the mechanistic alterations compound, ultimately resulting in the expression of spontaneous seizures. In C57BL/6J mice, evidence for this hypothesis is supported by flurothyl generalized seizure threshold data showing that repeated flurothyl-induced seizures lead to decreased generalized seizure thresholds. In order for the decreased generalized seizure thresholds to become permanent, C57BL/6J mice require eight generalized flurothyl seizures (Samoriski and Applegate, 1997).

There is increased interest in observations that epilepsy can sometimes enter remission without treatment (Sander, 1993; Sander and Shorvon, 1996; Kwan and Sander, 2004; Berg et al., 2006; Callaghan et al., 2007; Luciano and Shorvon, 2007). However, there is very little known as to why this can occur in humans. This is due in part to the lack of an appropriate animal model that develops spontaneous seizures with remission. To our knowledge, the use of the repeated flurothyl model in $\mathrm{C} 57 \mathrm{BL} / 6 \mathrm{~J}$ mice is the first that could allow for the mechanistic study of spontaneous seizure remission. It is interesting that, while C57BL/6J mice have remission of their seizures over the course of a 1 month recording period, DBA/2J mice do not appear to follow this same course. We propose that genetic factors influencing seizure remission in the repeated flurothyl model can be identified and subsequently evaluated in humans to predict and understand seizure remission. Studies are currently underway attempting to elucidate what these genetic differences could be and the signaling pathways that may be involved. Last, gene expression changes have been observed in C57BL/6J mice over the course of the flurothyl induction period (Kadiyala et al., 2015), which could indicate the possibility of flurothyl-induced and/or seizure-induced changes in gene expression contributing to the transient nature of spontaneous seizures in C57BL/6J mice. Genes that were shown to change with flurothyl-induced seizures were similar to what has been observed in electrical kindling models (Christensen et al., 2010; Kadiyala et al., 2015). This suggests that seizure remission could be due to the seizures and not an effect of flurothyl itself. However, more experiments are required to determine whether this is the case, for instance, by determining whether mice exposed to repeated seizures induced by pentylenetetrazol, or other $\mathrm{GABA}_{\mathrm{A}}$ antagonists, develop spontaneous seizures with remission. Moreover, increasing the number of flurothyl induction trials with either $10 \%$ or $100 \%$ flurothyl might also result in a permanence of spontaneous seizures, particularly since spontaneous seizures were observed in some of the animals 6 weeks following the $100 \%$ flurothyl induction. Such experiments would help to resolve whether the induction phase seizures are driving the spontaneous seizures observed in this flurothyl model or whether the emergence of the spontaneous seizure phenotype is due to changes in gene expression specific to flurothyl pharmacology. Understanding why seizures remit could have important implications for understanding epilepsy, since elucidating the mechanisms that are involved in seizure remission maylead to the development of novel antiepileptic treatments that may directly impact an eventual cure for epilepsy.

Our results in C57BL/6J mice suggest that on flurothyl rechallenge 1 month following the last flurothyl-induced seizure, mice 
having spontaneous seizures develop a more complex forebrain $\rightarrow$ brainstem seizure, whereas suppression of these spontaneous seizures with valproate prevented the evolution of forebrain $\rightarrow$ brainstem seizures. This indicates that the spontaneous seizures could be driving the evolution of the more complex seizure type (a forebrain $\rightarrow$ brainstem seizure). Studies are currently underway to determine whether spontaneous seizures drive a decrease in brainstem seizure thresholds of C57BL/6J mice that then allows for enhanced propagation of ictal discharge from the forebrain to the brainstem, possibly accounting for the expression of forebrain $\rightarrow$ brainstem seizures. Understanding the mechanisms underlying these observations may offer insights into why some patients develop complex seizure manifestations over time.

Overall, the repeated flurothyl model induces spontaneous seizures using "mild" seizure induction conditions (i.e., $<4 \mathrm{cu}-$ mulative minutes of induced seizure expression) and provides another tool for understanding the mechanisms of spontaneous seizure development (and remission). These spontaneous seizures are induced with minimal neuronal damage, which could greatly enhance our ability to define the basis for the initiation and remission of spontaneous seizures. This model of epilepsy adds to the collection of seizure models available and may help advance our understanding of the pathophysiological mechanisms underlying epilepsy and why seizures can enter remission, leading to the development of effective therapies to alleviate patient suffering due to chronic epilepsy.

\section{References}

Adler MW (1975) Pharmacology of flurothyl: laboratory and clinical applications. In: Current developments in psychopharmacology (Essman WB, Valzelli L, eds), pp 30-61. New York: Spectrum.

Adler MW, Sagel S, Kitagawa S, Segawa T, Maynert EW (1967) The effects of repeated flurothyl-induced seizures on convulsive thresholds and brain monoamines in rats. Arch Int Pharmacodyn 170:12-21. Medline

Applegate CD, Samoriski GM, Ozduman K (1997) Effects of valproate, phenytoin, and MK-801 in a novel model of epileptogenesis. Epilepsia 38: 631-636. CrossRef Medline

Berg AT, Vickrey BG, Testa FM, Levy SR, Shinnar S, DiMario F, Smith S (2006) How long does it take for epilepsy to become intractable? A prospective investigation. Ann Neurol 60:73-79. CrossRef Medline

Borges K, Gearing M, McDermott DL, Smith AB, Almonte AG, Wainer BH, Dingledine R (2003) Neuronal and glial pathological changes during epileptogenesis in the mouse pilocarpine model. Exp Neurol 182:21-34. CrossRef Medline

Browning RA, Nelson DK (1986) Modification of electroshock and pentylenetetrazol seizure patterns in rats after precollicular transections. Exp Neurol 93:546-556. CrossRef Medline

Callaghan BC, Anand K, Hesdorffer D, Hauser WA, French JA (2007) Likelihood of seizure remission in an adult population with refractory epilepsy. Ann Neurol 62:382-389. CrossRef Medline

Cavalheiro EA, Leite JP, Bortolotto ZA, Turski WA, Ikonomidou C, Turski L (1991) Long-term effects of pilocarpine in rats: structural damage of the brain triggers kindling and spontaneous recurrent seizures. Epilepsia 32: 778-782. CrossRef Medline

Chopde CT, Brahmankar DM, Shripad VN (1977) Neurochemical aspects of ethanol dependence and withdrawal reactions in mice. J Pharmacol Exp Ther 200:314-319. Medline

Christensen KV, Leffers H, Watson WP, Sánchez C, Kallunki P, Egebjerg J (2010) Levetiracetam attenuates hippocampal expression of synaptic plasticity-related immediate early and late response genes in amygdalakindled rats. BMC Neurosci 11:9. CrossRef Medline

Dolenz BJ (1967) Flurothyl (Indoklon) side effects. Am J Psychiatry 123: 1453-1455. CrossRef Medline

Fink M (2014) The seizure, not electricity, is essential in convulsive therapy: the flurothyl experience. J ECT 30:91-93. CrossRef Medline

Foldvary-Schaefer N, Grigg-Damberger M (2009) Sleep and epilepsy. Semin Neurol 29:419-428. CrossRef Medline
Gallagher BB (1969) Seizure threshold and hexafluorodiethyl ether in brain tissue. Biochem Phamacol 18:542-544. CrossRef

Goodman JH (1998) Experimental models of status epilepticus. In: Neuropharmacology methods in epilepsy research (Peterson SL, Albertson TE, eds), pp 95-125. Boca Raton, FL: CRC.

Kadiyala SB, Papandrea D, Tuz K, Anderson TM, Jayakumar S, Herron BJ, Ferland RJ (2015) Spatiotemporal differences in the c-fos pathway between C57BL/6J and DBA/2J mice following flurothyl-induced seizures: a dissociation of hippocampal Fos from seizure activity. Epilepsy Res 109: 183-196. CrossRef Medline

Kosobud A, Crabbe JC (1986) Ethanol withdrawal in mice bred to be genetically prone or resistant to ethanol withdrawal seizures. J Pharmacol Exp Ther 238:170-177. Medline

Krantz JC Jr, Truitt EB Jr, Ling AS, Speers L (1957) Anesthesia. LV. The pharmacologic response to hexafluorodiethyl ether. J Pharmacol Exp Ther 121:362-368. Medline

Krasowski MD (2000) Differential modulatory actions of the volatile convulsant flurothyl and its anesthetic isomer at inhibitory ligand-gated ion channels. Neuropharmacology 39:1168-1183. CrossRef Medline

Kreindler A, Zuckermann E, Steriade M, Chimion D (1958) Electroclinical features of convulsions induced by stimulation of brain stem. J Neurophysiol 21:430-436. Medline

Krishna V, Sammartino F, King NK, So RQ, Wennberg R (2016) Neuromodulation for epilepsy. Neurosurg Clin N Am 27:123-131. CrossRef Medline

Kwan P, Brodie MJ (2000) Early identification of refractory epilepsy. N Engl J Med 342:314-319. CrossRef Medline

Kwan P, Sander JW (2004) The natural history of epilepsy: an epidemiological view. J Neurol Neurosurg Psychiatry 75:1376-1381. CrossRef Medline

Leite JP, Bortolotto ZA, Cavalheiro EA (1990) Spontaneous recurrent seizures in rats: an experimental model of partial epilepsy. Neurosci Biobehav Rev 14:511-517. CrossRef Medline

Lemos T, Cavalheiro EA (1995) Suppression of pilocarpine-induced status epilepticus and the late development of epilepsy in rats. Exp Brain Res 102:423-428. Medline

Lindsten H, Stenlund H, Forsgren L (2001) Remission of seizures in a population-based adult cohort with a newly diagnosed unprovoked epileptic seizure. Epilepsia 42:1025-1030. Medline

Luciano AL, Shorvon SD (2007) Results of treatment changes in patients with apparently drug-resistant chronic epilepsy. Ann Neurol 62:375-381. CrossRef Medline

Mazzuferi M, Kumar G, Rospo C, Kaminski RM (2012) Rapid epileptogenesis in the mouse pilocarpine model: video-EEG, pharmacokinetic and histopathological characterization. Exp Neurol 238:156-167. CrossRef Medline

Papandrea D, Anderson TM, Herron BJ, Ferland RJ (2009) Dissociation of seizure traits in inbred strains of mice using the flurothyl kindling model of epileptogenesis. Exp Neurol 215:60-68. CrossRef Medline

Paxinos G, Franklin KBJ (2001) The mouse brain in stereotaxic coordinates. San Diego: Academic.

Perry MS, Duchowny M (2013) Surgical versus medical treatment for refractory epilepsy: outcomes beyond seizure control. Epilepsia 54:2060 2070. CrossRef Medline

Pitkänen A, Schwartzkroin PA, Moshé SL (2006) Models of seizures and epilepsy. Burlington, MA: Elsevier Academic.

Racine RJ (1972) Modification of seizure activity by electrical stimulation. II. Motor seizure. Electroencephalogr Clin Neurophysiol 32:281-294. CrossRef Medline

Samoriski GM, Applegate CD (1997) Repeated generalized seizures induce time-dependent changes in the behavioral seizure response independent of continued seizure induction. J Neurosci 17:5581-5590. Medline

Sander JW (1993) Some aspects of prognosis in the epilepsies: a review. Epilepsia 34:1007-1016. CrossRef Medline

Sander JW, Shorvon SD (1996) Epidemiology of the epilepsies. J Neurol Neurosurg Psychiatry 61:433-443. CrossRef Medline

Schachter SC (2016) Antiseizure drugs: mechanism of action, pharmacology, and adverse effects. In: UptoDate (Post TW, Rutgeerts P, Grover S, eds). Wellesley, MA: UpToDate. E-book available at http://www.uptodate.com/contents/ antiseizure-drugs-mechanism-of-action-pharmacology-and-adverse-effects.

Shibley H, Smith BN (2002) Pilocarpine-induced status epilepticus results in mossy fiber sprouting and spontaneous seizures in C57BL/6 and CD-1 mice. Epilepsy Res 49:109-120. CrossRef Medline 